\title{
Comparative Study of the Chemical Constituents and Bioactivities of the Extracts from Fruits, Leaves and Root Barks of Lycium barbarum
}

\author{
Xiao Xiao ${ }^{1,2, \dagger}$, Wei Ren ${ }^{1,2,+}\left(\mathbb{D}\right.$, Nan Zhang ${ }^{1,2}$, Tao Bing ${ }^{1,2} \mathbb{D}^{\text {, Xiangjun Liu }}{ }^{1,2}$, Zhenwen Zhao ${ }^{1,2, *}$ \\ and Dihua Shangguan 1,2,* \\ 1 Beijing National Laboratory for Molecular Sciences, Key Laboratory of Analytical Chemistry for Living \\ Biosystems, CAS Research/Education Center for Excellence in Molecular Sciences, Institute of Chemistry, \\ Chinese Academy of Sciences, Beijing 100190, China; xiaoxiao@iccas.ac.cn (X.X.); \\ renwei1991@iccas.ac.cn (W.R.); hszhang@iccas.ac.cn (N.Z.); bingtao@iccas.ac.cn (T.B.); xjliu@iccas.ac.cn (X.L.) \\ 2 University of the Chinese Academy of Sciences, Beijing 100049, China \\ * Correspondence: zhenwenzhao@iccas.ac.cn (Z.Z.); sgdh@iccas.ac.cn (D.S.) \\ + These authors contributed equally to this work.
}

Received: 1 April 2019; Accepted: 19 April 2019; Published: 22 April 2019

\begin{abstract}
The fruits, leaves and root barks of L. barbarum plant are widely used as functional foods and as ingredients in traditional Chinese prescriptions and patent medicines. They are considered to have different pharmacological activities and health benefits because of their diverse constituents. Here, the chemical constituents of the extracts from fruits, leaves and root barks of L. barbarum were compared by ultra-high performance liquid chromatography coupled with high resolution mass spectrometry (UPLC-HR-MS). A total of 131 compounds were identified and seven of them were quantified. Among them, 98, 28 and 35 constituents were detected in fruits, leaves and root barks respectively. Dicaffeoylspermidine/spermine derivatives were the most detected compounds (74/131); among them, dicaffeoylspermine isomers and propionyl-dicaffeoylspermidine were found in root barks in very large amounts (e.g., kukoamine $B=10.90 \mathrm{mg} / \mathrm{g}$ dry powder); dicaffeoyl-spermidine isomers were detected in fruits/leaves in a high amount, and many of their glycosylated derivatives were mainly detected in fruits. In addition, six saponins from L. barbarum fruits were reported for the first time, and 5,6-dihydrosolasonine was reported for the first time in plants. The activity assays showed that the root bark extract possessed the strongest antioxidative activity and cytotoxicity, which was presumed due to the large amount of dicaffeoylspermine/spermidines in root barks. Fourteen potential bioactive components from fruits were identified by a target cell-based screening method. These results will help to understand the different biological activities of these three parts of L. barbarum plant and will benefit the discovery of new functional components.
\end{abstract}

Keywords: chemical compounds; antioxidative activities; organs of Lycium barbarum; UPLC-MS; comparative study

\section{Introduction}

Lycium barbarum L. (L. barbarum), known as "goji" or Chinese wolfberry, belongs to the family of Solanaceae and is widely cultivated in China. Three parts of L. barbarum plant including fruits, leaves and root barks, have been used as functional foods and traditional Chinese medicinal herbs in China for centuries [1-3] and nowadays are being widely consumed all over the world. The fruits (goji berries, Chinese name: gouqizi) are reported to have multiple effects, such as anti-aging, neuroprotection, anti-fatigue, hypoglycemic, antiproliferative activity and cytoprotection, immunomodulation and antioxidant properties [3-5] and are being most widely used in foods and traditional medicines. The 
leaves, called "tianjingcao" in traditional Chinese medicine, have the benefits of alleviating mineral deficiency, combating heat distress, quenching thirst, dispelling wind, and enhancing eyesight, and have been widely used as tea, vegetables and medicines [6]. The root barks (Lycii Cortex Radicis, Chinese name: digupi or jikoppi) are officially listed in the Chinese Pharmacopoeia for the treatment of diabetes mellitus, night sweats, coughs, hematemesis, hypertension, and ulcers [7,8]. These three parts are considered to have different pharmacological activities and health benefits, and are widely used in different prescriptions and traditional Chinese patent medicines. The different biological activities of the three parts of L. barbarum plant are attributed to their different functional components. Many studies have identified and even quantified various chemical components in the three parts of Lycium genus plants individually, especially in fruits, including polysaccharides, peptide, alkaloids, flavonoids, terpenes, organic acids, lignans, phenolic amides, carotenoids, etc [3,9-11]. For example, Patsilinakos et al. have studied the carotenoid content in goji berries cultivated in Italy, evaluating the differences among varieties, harvesting periods, seasons, and extracting procedures by colorimetric and high performance liquid chromatograph-diode array detector (HPLC-DAD) analyses [12]. Inbaraj et al. have identified a total of 52 phenolic acids and flavonoids in Lycium barbarum Linnaeus by HPLC-DAD coupled electrospray ionization mass spectrometry (HPLC-DAD-ESI-MS) [13]. Mocan et al. have quantified eight phenolic acids and eleven flavonoids in L. barbarum and Lycium chinense Mill leaves [14]. However, to the best of our knowledge, few study has compared the difference of the chemical components of these three parts. The comparative study of the chemical components will help to understand the unique biological activities of these three parts and demonstrate their potential functional components.

Because of the excellent physical separation capability of UPLC, and the powerful identification ability of high-resolution mass spectrometry (HR-MS), UPLC coupled HR-MS (UPLC-HR-MS) has been extensively used in systematically identifying and quantifying components in complex samples [15]. For example, Patras et al. used UPLC-HR-MS to profile and quantify the regioisomeric caffeoyl glucoses in goji berry fruits [16]. Mocan et al. have employed UPLC coupled quadrupole-time of flight mass spectrometer (UPLC-QTOF-MS) to study the bioactive constituents of two Romanian Goji (L. barbarum) berries cultivars and evaluated their antioxidant and enzyme inhibitory properties [17]. Recently, UPLC coupled hybrid triple quadrupole linear ion trap mass spectrometer (UPLC-Qtrap-MS) with targeted multiple reactions monitoring (MRM) mode shows high sensitivity, specificity, and selectivity in the simultaneous identification and quantitation of compounds in large concentration ranges and complex matrices [18].

The aim of this study is to compare the chemical constituents and the bioactivities of the ethanol/water $(70 / 30, v / v)$ extracts from the fruits, leaves and root barks of L. barbarum. A total of 131 compounds were identified by UPLC coupled Orbitrap mass spectrometry (UPLC-Orbitrap-MS). Five compounds in fruits, one compound in root barks and three compounds in leaves were quantified by UPLC-Qtrap-MS. The antioxidative activity of these extracts was evaluated in buffer and in cells, respectively, and the cytotoxicity of the extracts was tested. In addition, a compound database was constructed based on the chemical constituents of fruits extract, based on which, the potential bioactive components in fruits of L. barbarum were identified by the target cell-based screening method.

\section{Results and Discussion}

\subsection{Isolation and Identification of 5,6-dihydrosolasonine from Fruits}

In our preliminary experiment of the qualitative analysis of fruits extract by UPLC-HR-MS, two compounds displaying $[\mathrm{M}+\mathrm{H}]^{+}$ions at $\mathrm{m} / \mathrm{z} 884.5084$ and 886.5241 attracted our attention because they had not been previously reported in L. barbarum. The compound with $[\mathrm{M}+\mathrm{H}]^{+}$ion at $\mathrm{m} / \mathrm{z} 884.5084$ was assigned to the molecular formula of $\mathrm{C}_{45} \mathrm{H}_{74} \mathrm{O}_{16} \mathrm{~N}$, with a mass error of $1.13 \mathrm{ppm}$. Based on its fragmentation pattern, this compound was identified to be the spirosolane-type glycoalkaloid, solasonine [19], which was further confirmed by comparing its retention time in UPLC (Figure S1) and 
MS/MS fragmentation ions with a solasonine standard (Figure S2). The fragment ions at $\mathrm{m} / \mathrm{z} 722.4544$ $\left[\mathrm{M}+\mathrm{H}-\mathrm{C}_{6} \mathrm{H}_{10} \mathrm{O}_{5}\right]^{+}, 576.3957\left[\mathrm{M}+\mathrm{H}-\mathrm{C}_{6} \mathrm{H}_{10} \mathrm{O}_{5}-\mathrm{C}_{6} \mathrm{H}_{10} \mathrm{O}_{4}\right]^{+}, 414.3403\left[\mathrm{M}+\mathrm{H}-\mathrm{C}_{6} \mathrm{H}_{10} \mathrm{O}_{5}-\right.$ $\left.\mathrm{C}_{6} \mathrm{H}_{10} \mathrm{O}_{4}-\mathrm{C}_{6} \mathrm{H}_{10} \mathrm{O}_{5}\right]^{+}$, correspond to the successive losses of glucosyl, rhamnosyl and glucosyl. The production at $m / z 414.3403$ was ascribed to the aglycone ion of the steroidal glycoalkaloid. The fragment ion at $\mathrm{m} / \mathrm{z} 271.2054$ originates from the neutral loss (143 Da) of the E-ring and nitrogen-containing F-ring moiety from the ion at $m / z$ 414.3403. The ion at $m / z 253.1950$ was formed by the neutral loss of $\mathrm{H}_{2} \mathrm{O}$ from the ion at $m / z 271.2054$ (Figure S2). The compound with $[\mathrm{M}+\mathrm{H}]^{+}$ion at $m / z 886.5152$ was assigned a molecular formula of $\mathrm{C}_{45} \mathrm{H}_{76} \mathrm{O}_{16} \mathrm{~N}$ with a mass error of 0.75 ppm, which has two more hydrogens than solasonine. Furthermore, most of its fragmentation ions showed two Da higher (Figure $1,868.5034 ; 722.4475 ; 578.4109 ; 416.3563 ; 398.3408 ; 273.2205 ; 255.2102$ ) than those of solasonine (Figure S2, 866.4896; 720.4325; 576.3898; 414.3403; 396.3259; 271.2054; 253.1949), suggesting the loss of the same neutral fragments during the MS fragmentation (e.g., the sugar moiety).

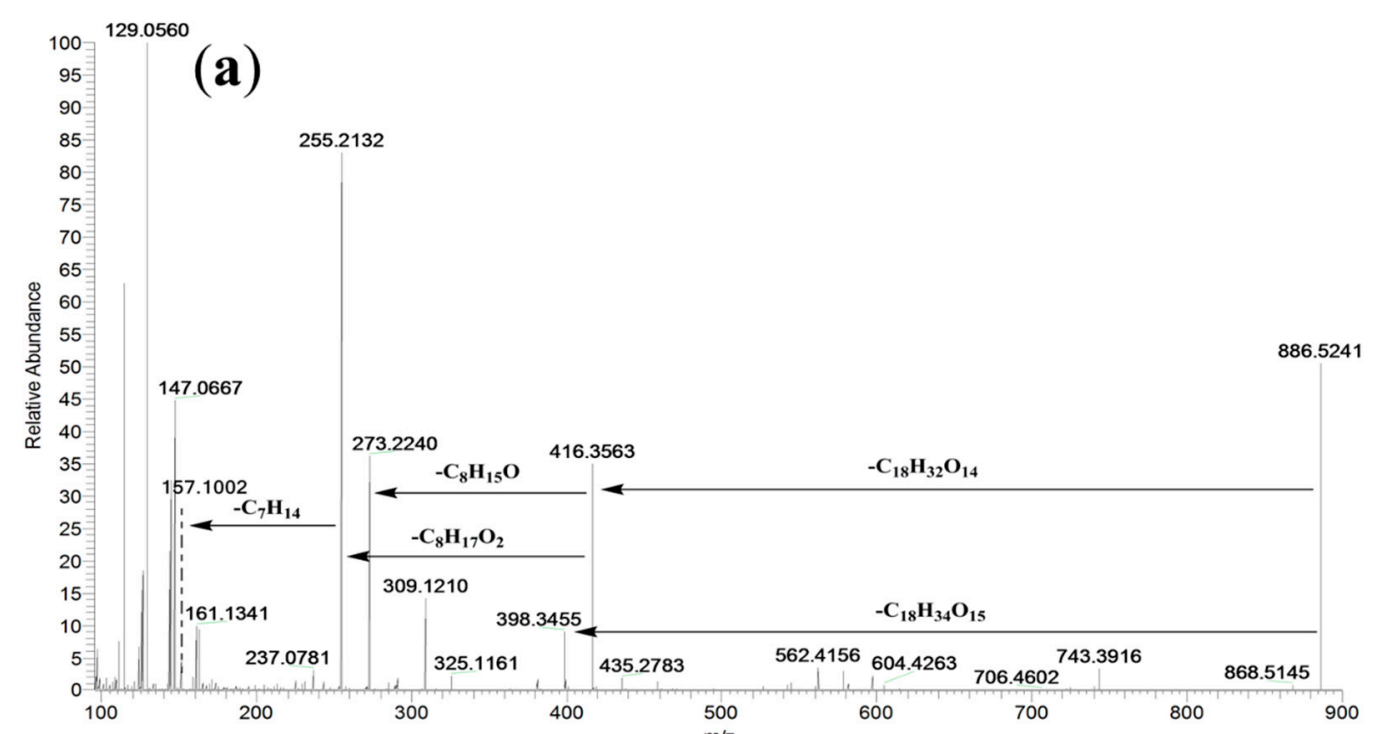

(b)

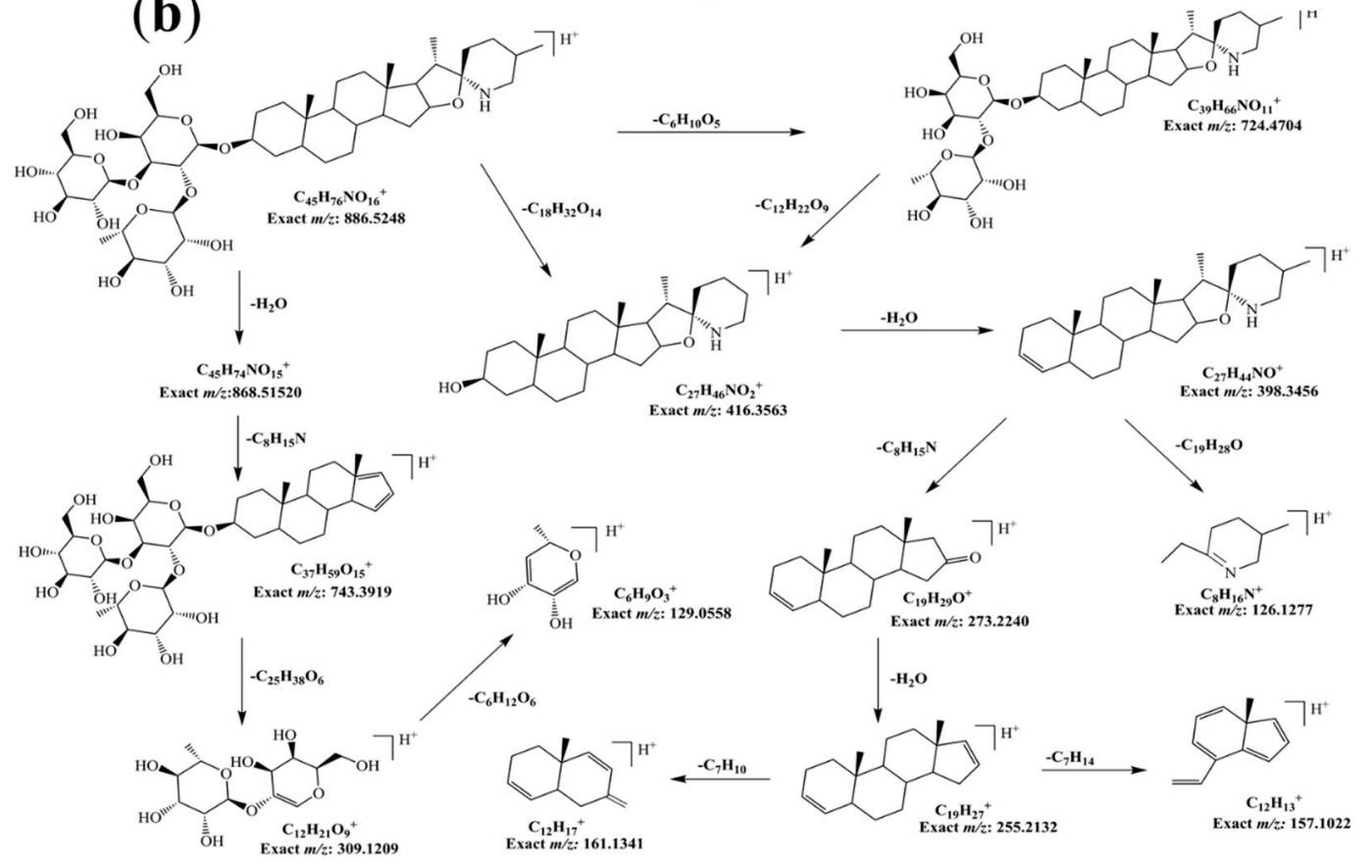

Figure 1. The HR-Orbitrap MS/MS spectrum (a) and proposed fragmentation pathway (b) of 5,6-dihydrosolasonine. 
This result suggests that the new compound has a similar structure to solasonine except for two more hydrogens on the aglycone. Based on the fragmentation ions of both compounds and the reported fragmentation pathway of solasonine [19], the new compound could be 5,6-dihydrosolasonine, which differs from solasonine by the C-C single bond at position 5,6 in the B-ring of the steroidal skeleton. The proposed aglycone of 5,6-dihydrosolasonine (named as soladulcidine) has been reported in other two glycoalkaloids (soladulcine A/B) isolated from Solanum dulcamara, which consist of chacotriose/lycotetraose and soladulcidine joined through a $\beta$-glycosidic bond [20]. The proposed fragmentation pathway for 5,6-dihydrosolasonine is shown in Figure 1. Furthermore, 5,6-dihydrosolasonine ( $20 \mathrm{mg}$, white powder, UV $\lambda_{\max }: 225 \mathrm{~nm}$ ) was isolated from dried fruit of L. barbarum $\left(5 \mathrm{~kg}\right.$ ) and characterized by MS and ${ }^{13} \mathrm{C}-\mathrm{NMR}$. The NMR data (Table S1) showed that the peaks at $140.6 \mathrm{ppm}$ and $121.4 \mathrm{ppm}$ in the ${ }^{13} \mathrm{C}-\mathrm{NMR}$ spectrum of solasonine disappeared in the ${ }^{13} \mathrm{C}-\mathrm{NMR}$ spectrum of 5,6-dihydrosolasonine, while two new peaks appeared at $43.06 \mathrm{ppm}$ and $28.77 \mathrm{ppm}$, which correspond to the change of the double bound to a C-C single bond at position 5,6 in the B-ring of the steroidal skeleton. In addition, soladulcidine $(22 R, 25 R)$ has a stereoisomer, tomatidine $(22 S, 25 S)$, the aglycone of $\alpha$-tomatine found in the stems and leaves of tomato plants. The ${ }^{13} \mathrm{C}$-NMR peak at $33.73 \mathrm{ppm}$ (C23) and $45.87 \mathrm{ppm}$ (C26) further confirmed that the aglycone of 5,6-dihydrosolasonine is soladulcidine [21].

Glycoalkaloids are nitrogen-containing steroidal glycosides, generally found in plants of the Solanaceae, such as tomato, potato, and aubergine [22]. Solasonine and solamargine are two major steroidal glycoalkaloids, which have been found in 200 Solanum species [23-25]. They are water soluble triglycosides with the same aglycone (solasodine) and different trioses (solatriose and chacotriose) [25]. Solasodine is one of the main aglycone of glycoalkaloids, and has been used as raw material for steroidal drugs. Although L. barbarum belongs to the Solanaceae family, solasonine has not been previously reported in L. barbarum. A ring E-opened dihydro-derivative of solasonine has been reported by Weissenberg et al. [26,27] which has the same molecular weight as 5,6-dihydrosolasonine, but should have different fragmentation ions from those shown in Figure 1. As far as we know, 5,6-dihydrosolasonine has not been previously identified in any plant. The isolated 5,6-dihydrosolasonine was used as a standard substance for the following quantitative study.

\subsection{Multi-Component Analysis of Extracts by UPLC-HR-MS}

Under the optimized UPLC-HR-MS experimental conditions, the accurate mass and composition for the precursor ions and product ions from the extracts of fruits, leaves and root barks were analyzed respectively using Xcalibur ${ }^{\mathrm{TM}} 3.0$ (Thermo Fisher) software in both positive and negative ionization modes. Internal calibration by infusion of a calibrant achieved a typical mass accuracy within $10 \mathrm{ppm}$. The identification of the compounds in extracts was performed based on the retention time, high resolution MS/MS data, isotope abundance, fragment product ions, literature data, databases (Reaxys, PubMed, Mass Bank, Chemspider, etc.) and standard substances. The fragmentation patterns (Figure 1 and Figures S2-S6) of seven standards were proposed based on their high resolution MS/MS spectra, which were further used to assist the identification of constituents in extracts. Finally, a total of 131 compounds were detected based on our analytical strategy (Figure 2). Based on their chemical structures, the detected compounds were classified into six groups, including phenylpropanoids, dicaffeoylspermidine/spermine derivatives, phenolic amides, flavones, saponins and others (Figure 2). The detailed information of the 131 compounds found in fruits, leaves and root barks of L. barbarum are presented in Table 1. 


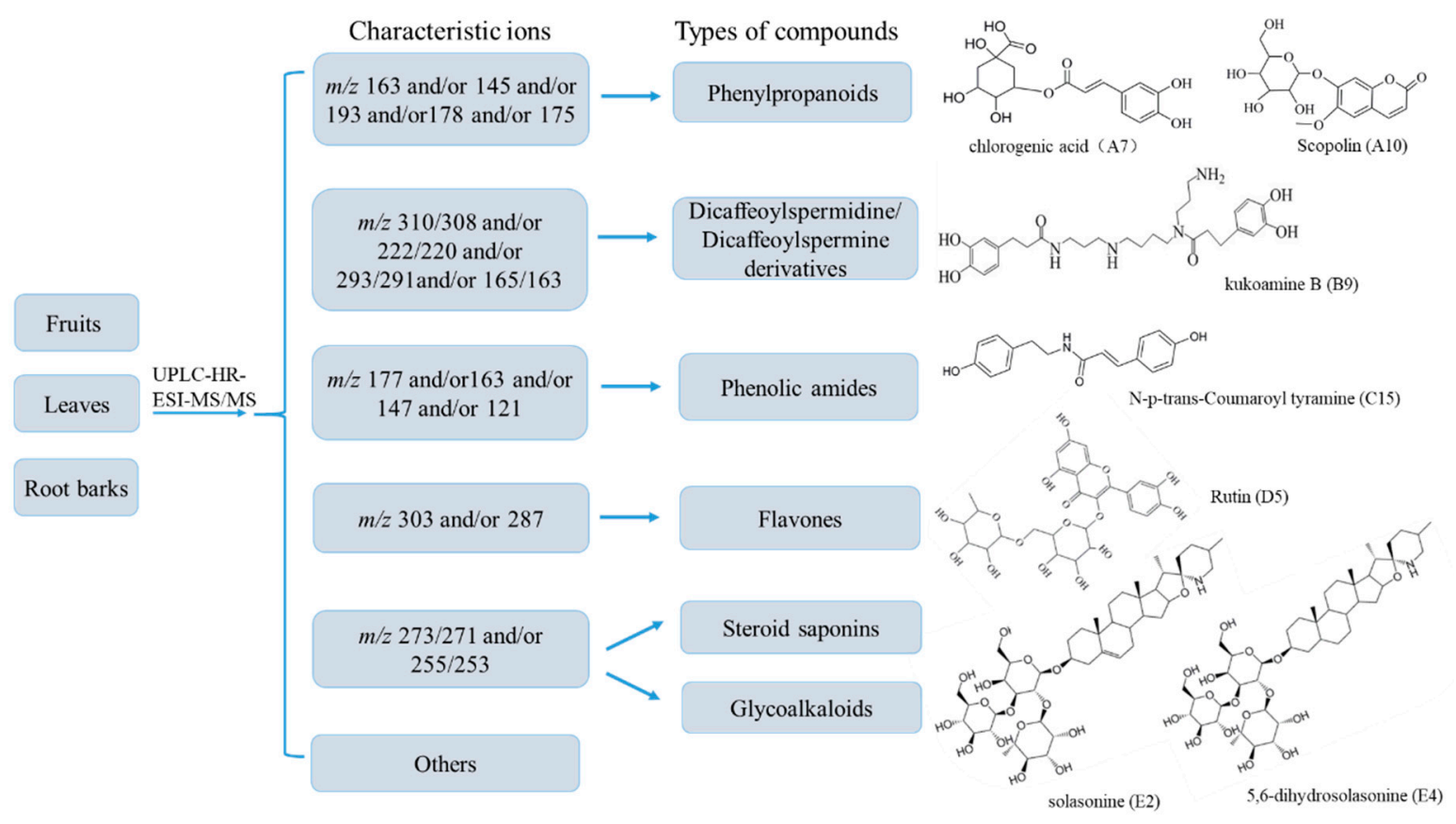

Figure 2. A diagram for rapid classification and tentative identification of chemical constituents in the extracts of fruits, leaves and root barks of L. barbarum by UPLC-HR-MS, and the structures of standard compounds in different classes.

Phenylpropanoid group included 13 phenylpropionate compounds containing caffeoyl, feruloyl, coumaroyl, sinapoyl or scopoletin group. These groups generate characteristic ions at $m / z 163$ and 145 for caffeoyl in the positive ion mode; $m / z 163$ and 145 for coumaroyl and $m / z 193$ and 175 for feruloyl in the negative ion mode; $m / z 193 / 191$ and 178/176 for scopoletin in positive/negative ion modes; and at $\mathrm{m} / z 185$ and 163 for sinapoyl in negative ion mode. For example, compound A7 with $[\mathrm{M}+\mathrm{H}]^{+}$ion at $\mathrm{m} / \mathrm{z} 355.1032\left(\mathrm{C}_{16} \mathrm{H}_{19} \mathrm{O}_{9}\right.$, Cal. 355.1023, mass error $\left.1.55 \mathrm{ppm}\right)$ was confirmed as chlorogenic acid by a standard. The ion at $\mathrm{m} / \mathrm{z} 163.0394$ was formed by the neutral loss of a quinoyl unit (193 Da) from the parent ion at $m / z 355.1032$ (Figure S3). Compound A1 displaying a $[\mathrm{M}-\mathrm{H}]^{-}$ion at $m / z 487.1485$ $\left(\mathrm{C}_{23} \mathrm{H}_{32} \mathrm{O}_{13}\right.$, Cal. 487.1425, mass error $\left.1.78 \mathrm{ppm}\right)$ was identified to be a lycibarbarphenylpropanoid $\mathrm{A}$ isomer; the key product with $\left[\mathrm{M}-\mathrm{H}-\mathrm{C}_{12} \mathrm{H}_{20} \mathrm{O}_{5}\right]^{-}$ion at $m / z 163.0403$ and $\left[\mathrm{M}-\mathrm{H}-\mathrm{C}_{12} \mathrm{H}_{20} \mathrm{O}_{5}-\right.$ $\mathrm{H}_{2} \mathrm{O}^{-}$ion at $\mathrm{m} / \mathrm{z} 145.0296$ indicated the existence of a coumaroyl moiety (Figure S8). Compound A10 with $[\mathrm{M}+\mathrm{H}]^{+}$ion at $m / z 355.1031\left(\mathrm{C}_{16} \mathrm{H}_{19} \mathrm{O}_{9}\right.$, Cal. 355.1023, mass error $\left.1.42 \mathrm{ppm}\right)$ and fragment ions at $\mathrm{m} / \mathrm{z} 193.0500$ and 178.0276 was identified to be scopolin as confirmed by a standard (Figure S4).

Dicaffeoylspermidine and dicaffeoylspermine derivatives are conjugates of caffeoyl groups and spermidine or spermine via amide bonds, which mainly contain characteristic fragments at $\mathrm{m} / \mathrm{z} 310 / 308$, $293 / 291,222 / 220$ or $165 / 163$. Compound B9 displaying a $[\mathrm{M}+\mathrm{H}]^{+}$ion at $m / z 531.3220\left(\mathrm{C}_{28} \mathrm{H}_{43} \mathrm{O}_{6} \mathrm{~N}_{4}, \mathrm{Cal}\right.$. 531.3177 , mass error $1.41 \mathrm{ppm}$ ) was confirmed to be the dicaffeoylspermine derivative kukoamine $\mathrm{B}$ by a standard (Figure S5). The fragment ion at $m / z 367.2736$ was formed by neutral loss of one caffeoyl unit, and the fragment ion at $m / z 165.0559$ was formed by further neutral loss of the spermine unit. In the same manner, compounds B1, B5, B7 and B10 were identified to be isomers of kukoamine B.

Compounds B2 and B3 displaying $[\mathrm{M}+\mathrm{H}]^{+}$ions at $\mathrm{m} / \mathrm{z} 855.4222\left(\mathrm{C}_{40} \mathrm{H}_{62} \mathrm{O}_{16} \mathrm{~N}_{4}\right.$, Cal. 855.4231, mass error $1.38 \mathrm{ppm}$ ) were identified to be two positional isomers of diglycosyl-caffeoyl spermine. Compounds B52 and B63 displaying $[\mathrm{M}+\mathrm{H}]^{+}$ion at $m / z 474.2585\left(\mathrm{C}_{25} \mathrm{H}_{35} \mathrm{O}_{6} \mathrm{~N}_{3}\right.$, Cal. 474.2598, mass error $2.78 \mathrm{ppm})$ and $472.2389\left(\mathrm{C}_{25} \mathrm{H}_{33} \mathrm{O}_{6} \mathrm{~N}_{3}\right.$, Cal. 472.2319, mass error $\left.1.64 \mathrm{ppm}\right)$ were dicaffeoylspermidines. Their fragment ions at $\mathrm{m} / \mathrm{z} 310.2120$ and 310.2119 were formed by neutral loss of one caffeoyl unit, and $\mathrm{m} / \mathrm{z} 165.0560$ and 163.0389 were formed by further neutral loss of the spermidine unit (Figures S9 and S10). 
Table 1. Compounds identified from fruits, leaves and root barks of L. barbarum by UPLC-Q-Orbitrap-MS/MS.

\begin{tabular}{|c|c|c|c|c|c|c|c|c|c|c|c|}
\hline No & $\mathrm{RT}^{1}$ & Formula & {$[\mathrm{M}+\mathrm{H}]^{+}$} & {$[\mathrm{M}-\mathrm{H}]^{-}$} & ppm & MS/MS Fragments ${ }^{2}$ & Identification & Ref. $^{3}$ & $\mathrm{~F}^{4}$ & $\mathrm{~L}^{5}$ & $R^{6}$ \\
\hline A1 & 8.70 & $\mathrm{C}_{21} \mathrm{H}_{29} \mathrm{O}_{13}$ & & 487.1485 & 1.78 & $324.8671 ; 303.7701 ; 163.0403 ; 145.0296 ; 119.0503$ & Lycibarbarphenylpropanoid A isomer & [28] & $\checkmark$ & & \\
\hline $\mathrm{A} 2$ & 9.72 & $\mathrm{C}_{21} \mathrm{H}_{29} \mathrm{O}_{13}$ & & 487.1492 & 1.22 & $398.1759 ; 229.0514 ; \mathbf{1 6 3 . 0 3 8 1} ; 145.0275 ; 119.0503$ & Lycibarbarphenylpropanoid A isomer & [28] & $\checkmark$ & & \\
\hline $\mathrm{A} 3$ & 10.44 & $\mathrm{C}_{22} \mathrm{H}_{30} \mathrm{O}_{14}$ & & 517.1583 & 0.72 & $334.8634 ; 235.0619 ; 193.0510 ; 175.0403 ; 160.0169 ; 134.0375$ & Lycibarbarphenylpropanoid $C$ isomer & [28] & $\checkmark$ & & \\
\hline $\mathrm{A} 4$ & 10.47 & $\mathrm{C}_{15} \mathrm{H}_{16} \mathrm{O}_{8}$ & & 325.0937 & 0.23 & 298.8872; 163.0398; $145.0276 ; 119.0497$ & p-coumaric acid O-glycosides & [16] & $\checkmark$ & $\checkmark$ & \\
\hline A5 & 15.45 & $\mathrm{C}_{16} \mathrm{H}_{18} \mathrm{O}_{9}$ & 355.1050 & 353.0854 & 1.34 & 308.9027; 285.0117; 181.0513; 163.0402; 145.0296; & chlorogenic acid isomer & [28] & & $\checkmark$ & \\
\hline A6 & 16.16 & $\mathrm{C}_{22} \mathrm{H}_{30} \mathrm{O}_{14}$ & & 517.1594 & 1.58 & $354.5810 ; 259.0620 ; 193.0510 ; 175.0403 ; 160.0169 ; 134.0375$ & Lycibarbarphenylpropanoid $\mathrm{C}$ isomer & [28] & $\checkmark$ & & \\
\hline A7 & 16.88 & $\mathrm{C}_{16} \mathrm{H}_{18} \mathrm{O}_{9}$ & 355.1050 & 353.0854 & 1.55 & 163.0395; 145.0289; 135.0445; 117.0339; 89.0390; & chlorogenic acid * & & & $\checkmark$ & \\
\hline A8 & 17.32 & $\mathrm{C}_{23} \mathrm{H}_{32} \mathrm{O}_{13}$ & & 515.1417 & 1.00 & 395.0988; 353.0877; 274.9858; 191.0557; 161.0242 & Lycibarbarphenylpropanoid F isomer & [28] & $\checkmark$ & $\checkmark$ & \\
\hline A9 & 18.78 & $\mathrm{C}_{23} \mathrm{H}_{32} \mathrm{O}_{13}$ & & 515.1422 & 1.24 & $323.0873 ; 274.9858 ; 191.0539 ; 161.0225$ & Lycibarbarphenylpropanoid $\mathrm{F}$ isomer & [28] & $\checkmark$ & $\checkmark$ & \\
\hline A10 & 18.87 & $\mathrm{C}_{16} \mathrm{H}_{18} \mathrm{O}_{9}$ & 355.1008 & 353.0855 & 1.42 & 303.0228; 193.0512; $178.0276 ; 134.0375$ & Scopolin * & & $\checkmark$ & & \\
\hline A11 & 18.92 & $\mathrm{C}_{27} \mathrm{H}_{36} \mathrm{O}_{18}$ & & 647.1873 & 1.34 & 485.2170; 323.1666; 191.0319; 176.0087; 161.0431; 148.0142 & lycibarbarcoumarin A & [28] & $\checkmark$ & & \\
\hline A12 & 20.42 & $\mathrm{C}_{16} \mathrm{H}_{18} \mathrm{O}_{9}$ & 355.1047 & 353.0854 & 1.34 & 285.0116; 193.0510; 163.0403; 145.0295; 123.1177 & chlorogenic acid isomer & [28] & & $\checkmark$ & \\
\hline A13 & 30.04 & $\mathrm{C}_{17} \mathrm{H}_{22} \mathrm{O}_{10}$ & & 385.1140 & 1.29 & $326.9597 ; 185.0199 ; \mathbf{1 6 3 . 0 3 8 1 ;} 119.0486$ & sinapate $4-O-\beta$-glucopyranoside & [29] & $\checkmark$ & $\checkmark$ & \\
\hline B1 & 17.87 & $\mathrm{C}_{28} \mathrm{H}_{42} \mathrm{O}_{6} \mathrm{~N}_{4}$ & 531.3170 & 529.3001 & 1.41 & $513.3074 ; 367.2724 ; 293.1855 ; 222.1123 ; 165.0546$ & kukoamine B ismoer & [30] & & $\checkmark$ & \\
\hline B2 & 18.48 & $\mathrm{C}_{40} \mathrm{H}_{62} \mathrm{O}_{16} \mathrm{~N}_{4}$ & 855.4222 & 853.4034 & 1.38 & $\begin{array}{c}\text { 693.3734; 529.3273; } 455.2405 ; 384.1668 ; 293.1871 ; \\
222.1121 ; 165.0545\end{array}$ & 2Glu-[kukoamine] ismoer & & $\checkmark$ & & \\
\hline B3 & 19.30 & $\mathrm{C}_{40} \mathrm{H}_{62} \mathrm{O}_{16} \mathrm{~N}_{4}$ & 855.4222 & 853.4034 & 1.38 & $\begin{array}{c}\text { 693.3693; 531.3206; } 455.2379 ; 384.1668 ; 293.1853 ; \\
222.1121 ; 165.0545\end{array}$ & 2Glu-[kukoamine] ismoer & & $\checkmark$ & & \\
\hline B4 & 19.48 & $\mathrm{C}_{46} \mathrm{H}_{72} \mathrm{O}_{21} \mathrm{~N}_{4}$ & 1017.4749 & 1015.4559 & 1.31 & 855.4216; 617.2904; 455.2379; 384.1645; 222.1122; 165.0557 & 3Glu-[kukoamine] ismoer & & $\checkmark$ & & \\
\hline B5 & 19.50 & $\mathrm{C}_{28} \mathrm{H}_{42} \mathrm{O}_{6} \mathrm{~N}_{4}$ & 531.3213 & 529.3011 & 1.41 & $513.3074 ; 367.2728 ; 293.1855 ; 222.1123 ; 165.0546$ & kukoamine B ismoer & & $\checkmark$ & & \\
\hline B6 & 19.59 & $\mathrm{C}_{34} \mathrm{H}_{52} \mathrm{O}_{11} \mathrm{~N}_{4}$ & 693.3698 & 691.3521 & 1.05 & $531.3168 ; 367.2730 ; 293.1855 ;$ 222.1122; $165.0560 ; 123.0440$ & Glu-[kukoamine] ismoer & & $\checkmark$ & & \\
\hline B7 & 20.28 & $\mathrm{C}_{28} \mathrm{H}_{42} \mathrm{O}_{6} \mathrm{~N}_{4}$ & 531.3165 & 529.3001 & 1.41 & $513.3103 ; 447.8046 ; 376.2692 ; 293.1879 ; 222.1140 ; 165.8764$ & kukoamine A & [30] & & & $\checkmark$ \\
\hline B8 & 20.60 & $\mathrm{C}_{46} \mathrm{H}_{72} \mathrm{O}_{21} \mathrm{~N}_{4}$ & 1017.4747 & 1015.4559 & 1.49 & 855.4216; $617.2901 ; 455.2379 ; 384.1642 ; 222.1121 ; 165.0552$ & 3Glu-[kukoamine] ismoer & & $\checkmark$ & & \\
\hline B9 & 20.65 & $\mathrm{C}_{28} \mathrm{H}_{42} \mathrm{O}_{6} \mathrm{~N}_{4}$ & 531.3220 & 529.3001 & 1.41 & $513.3074 ; 367.2712 ; 310.2152 ; 293.1855 ; 222.1123 ; 165.0545$ & kukoamine B* & & & & $\checkmark$ \\
\hline B10 & 21.02 & $\mathrm{C}_{28} \mathrm{H}_{42} \mathrm{O}_{6} \mathrm{~N}_{4}$ & 531.3199 & 529.3003 & 1. 40 & $\begin{array}{c}\text { 402.9773; 367.2716; 293.1880; } 222.1141 ; 193.0510 ; \\
\text { 165.0553; } 129.1395\end{array}$ & kukoamine B ismoer & {$[30]$} & $\checkmark$ & & \\
\hline B11 & 21.73 & $\mathrm{C}_{28} \mathrm{H}_{40} \mathrm{O}_{6} \mathrm{~N}_{4}$ & 529.3059 & 527.2831 & 1.13 & $472.2346 ; 367.2725 ; 293.1877 ; 222.1140 ; 163.0402$ & $\begin{array}{c}\text { Dihydrocaffeoyl quinonespermine } \\
\text { ismoer } 7\end{array}$ & [31] & & & $\checkmark$ \\
\hline B12 & 23.31 & $\mathrm{C}_{28} \mathrm{H}_{40} \mathrm{O}_{6} \mathrm{~N}_{4}$ & 529.3012 & 527.2847 & 1.72 & $511.2894 ; 455.2384 ; 384.1648 ; 293.1855 ;$ 220.0986; 163.0406 & Dihydrocaffeoyl quinonespermine ismoer & [31] & $\checkmark$ & $\checkmark$ & \\
\hline B13 & 23.83 & $\mathrm{C}_{43} \mathrm{H}_{63} \mathrm{O}_{21} \mathrm{~N}_{3}$ & 958.4016 & 956.3817 & 1.18 & 796.3486; 634.2960; $472.2396 ; 310.2119 ; \mathbf{2 2 0 . 0 9 6 6} ; 163.0388$ & Glu-[lycibarbarspermidine F] isomer & & $\checkmark$ & & \\
\hline B14 & 24.41 & $\mathrm{C}_{37} \mathrm{H}_{51} \mathrm{O}_{16} \mathrm{~N}_{3}$ & 794.3339 & 792.3145 & 0.36 & $632.2804 ; 470.2540 ; 382.1489 ; 220.0965 ; 163.0388$ & [lycibarbarspermidine $\mathrm{O}$ ] isomer & [32] & $\checkmark$ & & \\
\hline B15 & 24.41 & $\mathrm{C}_{43} \mathrm{H}_{65} \mathrm{O}_{21} \mathrm{~N}_{3}$ & 960.4161 & 958.3975 & 2.28 & 798.3622; 636.3112; $474.2588 ; 384.1645 ;$ 222.1122; 163.0402 & Glu-[lycibarbarspermidine M]ismoer & & $\checkmark$ & & \\
\hline B16 & 24.75 & $\mathrm{C}_{37} \mathrm{H}_{55} \mathrm{O}_{16} \mathrm{~N}_{3}$ & 798.3640 & 796.3453 & 1.80 & $636.3071 ; 474.2589 ; 384.1644 ; 220.0965 ; 163.0388$ & [lycibarbarspermidine $\mathrm{M}$ ] isomer & [32] & $\checkmark$ & & \\
\hline B17 & 24.78 & $\mathrm{C}_{37} \mathrm{H}_{53} \mathrm{O}_{16} \mathrm{~N}_{3}$ & 796.3492 & 794.3301 & 180 & 634.2957; $472.2431 ; 310.2126 ; 220.0965 ; 163.0398$ & [lycibarbarspermidine $\mathrm{F}]$ isomer & [32] & $\checkmark$ & & \\
\hline B18 & 24.80 & $\mathrm{C}_{31} \mathrm{H}_{43} \mathrm{O}_{11} \mathrm{~N}_{3}$ & 634.2959 & 632.2784 & 1.31 & $472.2431 ; 310.2122 ; 220.0966 ; 163.0390$ & lycibarbarspermidine $B$ isomer & [32] & $\checkmark$ & & \\
\hline B19 & 24.94 & $\mathrm{C}_{43} \mathrm{H}_{63} \mathrm{O}_{21} \mathrm{~N}_{3}$ & 958.4267 & 956.3817 & 1.82 & $796.3481 ; 634.2957 ; 472.2414 ; 310.2119 ; \mathbf{2 2 0 . 0 9 6 4} ; 163.0388$ & Glu-[lycibarbarspermidine $\mathrm{F}$ ] isomer & & $\checkmark$ & & \\
\hline $\mathrm{B} 20$ & 25.17 & $\mathrm{C}_{37} \mathrm{H}_{55} \mathrm{O}_{16} \mathrm{~N}_{3}$ & 798.3640 & 796.3453 & 1.89 & 636.3117; $474.2589 ; 384.1644 ; 222.1121 ; 165.0545$ & [lycibarbarspermidine $\mathrm{M}]$ isomer & [32] & $\checkmark$ & & \\
\hline B21 & 25.62 & $\mathrm{C}_{28} \mathrm{H}_{42} \mathrm{O}_{5} \mathrm{~N}_{4}$ & 515.3267 & & 7.61 & $\begin{array}{c}\text { 498.2995; } 367.2717 ; 293.7878 ; 277.1928 ; 222.1139 ; \\
165.0556 ; 129.1395\end{array}$ & Dihydrocaffeoyl spermine derivative & & & & $\checkmark$ \\
\hline B22 & 25.72 & & 529.3054 & 527.2847 & 7.16 & 458.2317; 367.2724; 291.1721; 220.0988; 163.0401 & Dihydrocaffeoyl quinonespermine ismoer & [31] & & $\checkmark$ & $\checkmark$ \\
\hline B23 & 25.72 & $\mathrm{C}_{28} \mathrm{H}_{40} \mathrm{O}_{6} \mathrm{~N}_{4}$ & 529.3059 & 527.2847 & 1.72 & $511.2894 ; 393.2533 ; 384.1649 ; 291.1745 ; 220.2846 ; 163.0422$ & Dihydrocaffeoyl quinonespermine ismoer & [31] & & $\checkmark$ & \\
\hline B24 & 25.77 & $\mathrm{C}_{43} \mathrm{H}_{63} \mathrm{O}_{21} \mathrm{~N}_{3}$ & 958.4016 & 956.3817 & 1.69 & $796.3489 ; 634.2968 ; 472.2392 ; 310.2118 ; 220.09656 ; 163.0383$ & Glu-[lycibarbarspermidine F] isomer & & $\checkmark$ & & \\
\hline B25 & 26.02 & $\mathrm{C}_{37} \mathrm{H}_{55} \mathrm{O}_{16} \mathrm{~N}_{3}$ & 798.3626 & 796.3453 & 3.63 & $636.3112 ; 474.2581 ; 384.1645 ; \mathbf{2 2 2 . 1 1 2 8} ; 165.0544$ & [lycibarbarspermidine $\mathrm{M}$ ] isomer & [32] & $\checkmark$ & & \\
\hline B26 & 26.10 & $\mathrm{C}_{37} \mathrm{H}_{53} \mathrm{O}_{16} \mathrm{~N}_{3}$ & 796.3409 & 794.3301 & 1.92 & 634.2957; $472.2431 ; 310.2121 ; \mathbf{2 2 0 . 0 9 6 5 ;} \mathbf{1 6 3 . 0 3 9 0}$ & [lycibarbarspermidine $\mathrm{F}]$ isomer & [32] & $\checkmark$ & & \\
\hline
\end{tabular}


Table 1. Cont.

\begin{tabular}{|c|c|c|c|c|c|c|c|c|c|c|c|}
\hline No & $\mathrm{RT}^{1}$ & Formula & {$[\mathbf{M}+\mathbf{H}]^{+}$} & {$[\mathrm{M}-\mathrm{H}]^{-}$} & ppm & MS/MS Fragments ${ }^{2}$ & Identification & Ref. $^{3}$ & $\mathrm{~F}^{4}$ & $L^{5}$ & $\mathbf{R}^{6}$ \\
\hline B27 & 26.36 & $\mathrm{C}_{43} \mathrm{H}_{65} \mathrm{O}_{21} \mathrm{~N}_{3}$ & 960.4125 & 958.3969 & 3.30 & 798.3621; 636.3112; 474.2590; 384.1646; 222.1121; 163.0404 & Glu-[lycibarbarspermidine M]ismoer & & $\checkmark$ & & \\
\hline B28 & 26.41 & $\mathrm{C}_{43} \mathrm{H}_{63} \mathrm{O}_{21} \mathrm{~N}_{3}$ & 958.4011 & 956.3817 & 1.63 & $\begin{array}{c}\text { 796.3483; 634.2957; } 472.2428 ; 398.1824 ; 310.2119 ; \\
\text { 220.0965; } 163.0388\end{array}$ & Glu-[lycibarbarspermidine F] isomer & & $\checkmark$ & & \\
\hline B29 & 26.44 & $\mathrm{C}_{37} \mathrm{H}_{55} \mathrm{O}_{16} \mathrm{~N}_{3}$ & 798.3609 & 796.3453 & 1.58 & $636.3071 ; 474.2585 ; 384.16455 ; 222.1122 ; 163.0385$ & [lycibarbarspermidine $\mathrm{M}$ ] isomer & & $\checkmark$ & & \\
\hline B30 & 26.79 & $\mathrm{C}_{43} \mathrm{H}_{63} \mathrm{O}_{21} \mathrm{~N}_{3}$ & 958.4009 & 956.3817 & 1.82 & $\begin{array}{c}\text { 796.3530; 634.2994; } 472.2431 ; 382.1511 ; 310.2120 ; \\
\text { 220.0978; } 163.0398\end{array}$ & Glu-[lycibarbarspermidine F] isomer & & $\checkmark$ & & \\
\hline B31 & 27.02 & $\mathrm{C}_{31} \mathrm{H}_{43} \mathrm{O}_{11} \mathrm{~N}_{3}$ & 634.2964 & 632.2784 & 1.03 & $472.24500 ; 310.21201 ; 220.09660 ; 163.03981$ & lycibarbarspermidine B isomer & [32] & $\checkmark$ & & \\
\hline B32 & 27.03 & $\mathrm{C}_{31} \mathrm{H}_{41} \mathrm{O}_{11} \mathrm{~N}_{3}$ & 632.2814 & 630.2632 & 0.07 & $470.2284 ; 382.1489 ; 308.1962 ; 220.0965 ; 163.0388$ & lycibarbarspermidine $\mathrm{N}$ isomer & [32] & $\checkmark$ & & \\
\hline B33 & 27.23 & $\mathrm{C}_{43} \mathrm{H}_{63} \mathrm{O}_{21} \mathrm{~N}_{3}$ & 958.3870 & 956.3817 & 1.80 & $796.34717 ; 634.29457 ; 472.2424 ; 310.2124 ; \mathbf{2 2 0 . 0 9 6 4 ;} 163.0383$ & Glu-[lycibarbarspermidine F] isomer & & $\checkmark$ & & \\
\hline B34 & 27.42 & $\mathrm{C}_{43} \mathrm{H}_{65} \mathrm{O}_{21} \mathrm{~N}_{3}$ & 960.4155 & 958.3969 & 9.00 & $798.3629 ; 636.3112 ; 474.2588 ; 384.1645 ; 222.1121 ; 165.0404$ & Glu-[lycibarbarspermidine $\mathrm{M}$ ] ismoer & & $\checkmark$ & & \\
\hline B35 & 27.51 & $\mathrm{C}_{31} \mathrm{H}_{45} \mathrm{O}_{11} \mathrm{~N}_{3}$ & 636.3115 & 634.2941 & 2.00 & $474.2589 ; 384.1649 ; \mathbf{2 2 2 . 1 1 2 1} ; 165.0544$ & lycibarbarspermidine J & & $\checkmark$ & & \\
\hline B36 & 27.73 & $\mathrm{C}_{31} \mathrm{H}_{41} \mathrm{O}_{11} \mathrm{~N}_{3}$ & 632.2811 & 630.2632 & 0.50 & $470.2276 ; 382.1480 ; 308.1962 ; 220.0963 ; 163.0388$ & lycibarbarspermidine $\mathrm{N}$ isomer & & $\checkmark$ & & \\
\hline B37 & 27.85 & $\mathrm{C}_{37} \mathrm{H}_{51} \mathrm{O}_{16} \mathrm{~N}_{3}$ & 794.3337 & 792.3145 & 0.67 & $632.28010 ; 470.2540 ; 382.1489 ; 220.0965 ; 163.0388$ & [lycibarbarspermidine $\mathrm{O}$ ] isomer & & $\checkmark$ & & \\
\hline B38 & 27.91 & $\mathrm{C}_{43} \mathrm{H}_{63} \mathrm{O}_{21} \mathrm{~N}_{3}$ & 958.3870 & 956.3817 & 1.22 & $796.3475 ; 634.2955 ; 472.2420 ; 310.2120 ; 220.09654 ; 163.0388$ & Glu-[lycibarbarspermidine $\mathrm{F}$ ] isomer & & $\checkmark$ & & \\
\hline B39 & 28.04 & $\mathrm{C}_{37} \mathrm{H}_{53} \mathrm{O}_{16} \mathrm{~N}_{3}$ & 796.3495 & 794.3301 & 1.39 & $634.2959 ; 472.2431 ; 310.2122 ; 220.0966 ; 163.0391$ & [lycibarbarspermidine $\mathrm{F}]$ isomer & [32] & $\checkmark$ & & \\
\hline B40 & 28.16 & $\mathrm{C}_{43} \mathrm{H}_{61} \mathrm{O}_{21} \mathrm{~N}_{3}$ & 956.3857 & 954.3660 & 1.48 & $794.3328 ; 632.2972 ; 470.2267 ; 220.0965 ; \mathbf{1 6 3 . 0 3 8 7}$ & Glu-[lycibarbarspermidine $\mathrm{O}$ ] ismoer & & $\checkmark$ & & \\
\hline B41 & 28.30 & $\mathrm{C}_{43} \mathrm{H}_{65} \mathrm{O}_{21} \mathrm{~N}_{3}$ & 960.4146 & 958.3969 & 3.39 & $798.3626 ; 636.3109 ; 474.2587 ; 384.1645 ; 222.1122 ; 165.0404$ & Glu-[lycibarbarspermidine M]ismoer & & $\checkmark$ & & \\
\hline B42 & 28.57 & $\mathrm{C}_{31} \mathrm{H}_{43} \mathrm{O}_{11} \mathrm{~N}_{3}$ & 634.2960 & 632.2784 & 1.69 & $472.2424 ; 382.1488 ; 310.2118 ; 220.0969 ; 163.0388$ & lycibarbarspermidine B isomer & [32] & $\checkmark$ & & \\
\hline B43 & 28.64 & $\mathrm{C}_{29} \mathrm{H}_{44} \mathrm{O}_{6} \mathrm{~N}_{4}$ & 545.3377 & 543.3162 & 7.85 & $\begin{array}{c}527.3255 ; 432.0255 ; 322.2652 ; 293.1878 ; 236.1295 ; \\
\text { 222.1139; } 129.1396\end{array}$ & Dihydrocaffeoyl spermine derivative & & & & $\checkmark$ \\
\hline B44 & 28.77 & $\mathrm{C}_{41} \mathrm{H}_{57} \mathrm{O}_{20} \mathrm{~N}_{3}$ & 912.3596 & 910.3403 & 1.38 & $750.3066 ; 634.2957 ; 472.2431 ; 310.2121 ; 220.0965 ; 163.0398$ & Dihydrocaffeoyl spermidine derivative & & $\checkmark$ & & \\
\hline B45 & 28.83 & $\mathrm{C}_{29} \mathrm{H}_{44} \mathrm{O}_{6} \mathrm{~N}_{4}$ & 545.3377 & 543.3162 & 7.97 & $\begin{array}{c}527.3264 ; 381.2884 ; 307.4034 ; 293.1878 ; 222.1139 ; \\
165.14386 ; 129.1396\end{array}$ & Dihydrocaffeoyl spermine derivative & & & & $\checkmark$ \\
\hline B46 & 29.06 & $\mathrm{C}_{49} \mathrm{H}_{73} \mathrm{O}_{26} \mathrm{~N}_{3}$ & 1120.4537 & 1118.4336 & 1.58 & 958.3557; 796.3481; 634.2957; 310.2118; 220.0965; $\mathbf{1 6 3 . 0 4 0 2}$ & 2Glu-[lycibarbarspermidine F] ismoer & & $\checkmark$ & & \\
\hline B47 & 29.11 & $\mathrm{C}_{41} \mathrm{H}_{57} \mathrm{O}_{20} \mathrm{~N}_{3}$ & 912.3597 & 910.3403 & 1.25 & $750.3068 ; 634.2960 ; 472.2431 ; 310.2121 ; 220.0965 ; 163.0398$ & Dihydrocaffeoyl spermidine derivative & & $\checkmark$ & & \\
\hline B48 & 29.16 & $\mathrm{C}_{31} \mathrm{H}_{43} \mathrm{O}_{11} \mathrm{~N}_{3}$ & 634.2959 & 632.2780 & 1.79 & 617.2727; 558.1018; $472.2414 ; 310.2120 ; 220.0966 ; 163.0388$ & lycibarbarspermidine B isomer & [32] & $\checkmark$ & $\checkmark$ & \\
\hline B49 & 29.23 & $\mathrm{C}_{31} \mathrm{H}_{41} \mathrm{O}_{11} \mathrm{~N}_{3}$ & 632.2813 & 630.2630 & 0.12 & $\begin{array}{c}\text { 604.2890; 587.2619; } 470.2543 ; 382.1489 ; 308.1963 ; \\
\text { 220.0965; } 163.0388\end{array}$ & lycibarbarspermidine $\mathrm{N}$ isomer & & $\checkmark$ & & \\
\hline B50 & 29.29 & $\mathrm{C}_{49} \mathrm{H}_{73} \mathrm{O}_{26} \mathrm{~N}_{3}$ & 1120.4536 & 1118.4336 & 1.69 & $\begin{array}{c}\text { 958.3558; 796.34814; 634.2957; } 472.2396 ; 310.2118 ; \\
\mathbf{2 2 0 . 0 9 6 5 ; ~ 1 6 3 . 0 4 1 2}\end{array}$ & 2Glu-[lycibarbarspermidine F] ismoer & & $\checkmark$ & & \\
\hline B51 & 29.32 & $\mathrm{C}_{37} \mathrm{H}_{51} \mathrm{O}_{16} \mathrm{~N}_{3}$ & 794.3336 & 792.3145 & 0.82 & $632.2830 ; 470.2540 ; 382.1489 ; 220.0966 ; \mathbf{1 6 3 . 0 3 8 9}$ & [lycibarbarspermidine $\mathrm{O}$ ] isomer & & $\checkmark$ & & \\
\hline B52 & 29.40 & $\mathrm{C}_{25} \mathrm{H}_{35} \mathrm{O}_{6} \mathrm{~N}_{3}$ & 474.2585 & 472.2435 & 2.79 & $457.2319 ; 310.2120 ; 222.1121 ; 165.0544 ; 123.0438$ & $N^{1}-N^{10}$ dihydrocaffeoyl spermidine & {$[31,33]$} & $\checkmark$ & $\checkmark$ & $\checkmark$ \\
\hline B53 & 29.44 & $\mathrm{C}_{41} \mathrm{H}_{57} \mathrm{O}_{20} \mathrm{~N}_{3}$ & 912.3593 & 910.3403 & 1.71 & $750.3066 ; 634.2957 ; 448.1598 ; 310.2121 ; \mathbf{2 2 0 . 0 9 6 5 ;} 163.0398$ & Dihydrocaffeoyl spermidine derivative & & $\checkmark$ & & \\
\hline B54 & 29.58 & $\mathrm{C}_{49} \mathrm{H}_{73} \mathrm{O}_{26} \mathrm{~N}_{3}$ & 1120.4535 & 1118.4336 & 1.80 & $958.3557 ; 796.3481 ; 634.2957 ; 310.2115 ; \mathbf{2 2 0 . 0 9 6 5 ;} \mathbf{1 6 3 . 0 4 1 2}$ & 2Glu-[lycibarbarspermidine F] ismoer & & $\checkmark$ & & \\
\hline B55 & 29.59 & $\mathrm{C}_{31} \mathrm{H}_{43} \mathrm{O}_{11} \mathrm{~N}_{3}$ & 634.2960 & 632.2784 & 1.70 & $513.0655 ; 472.2426 ; 310.2116 ; 222.1120 ; 163.0388$ & lycibarbarspermidine B isomer & & $\checkmark$ & & \\
\hline B56 & 29.60 & $\mathrm{C}_{31} \mathrm{H}_{41} \mathrm{O}_{11} \mathrm{~N}_{3}$ & 632.2805 & 630.2630 & 1.42 & $496.2304 ; 470.2259 ; 382.1489 ; 308.1962 ; \mathbf{2 2 0 . 0 9 6 5 ;} 163.0388$ & lycibarbarspermidine $\mathrm{N}$ isomer & & $\checkmark$ & & \\
\hline B57 & 29.64 & $\mathrm{C}_{41} \mathrm{H}_{57} \mathrm{O}_{20} \mathrm{~N}_{3}$ & 912.3593 & 910.3403 & 1.71 & $750.3065 ; 588.2751 ; 382.1488 ; 310.2121 ; \mathbf{2 2 0 . 0 9 6 4 ;} 163.0398$ & Dihydrocaffeoyl spermidine derivative & & $\checkmark$ & & \\
\hline B58 & 29.71 & $\mathrm{C}_{37} \mathrm{H}_{53} \mathrm{O}_{16} \mathrm{~N}_{3}$ & 796.3480 & 794.3300 & 2.31 & $634.2957 ; 472.2431 ; 310.2121 ; 220.0965 ; 163.0390$ & [lycibarbarspermidine $\mathrm{F}]$ isomer & & $\checkmark$ & & \\
\hline B59 & 29.71 & $\mathrm{C}_{37} \mathrm{H}_{51} \mathrm{O}_{16} \mathrm{~N}_{3}$ & 794.3331 & 792.3145 & 1.44 & $632.2804 ; 470.2540 ; 308.1962 ; 220.0964 ; 163.0388$ & [lycibarbarspermidine $\mathrm{O}]$ isomer & & $\checkmark$ & & \\
\hline $\mathrm{B} 60$ & 29.74 & $\mathrm{C}_{35} \mathrm{H}_{47} \mathrm{O}_{15} \mathrm{~N}_{3}$ & 750.3065 & 748.2889 & 1.11 & $588.2747 ; 472.2429 ; 310.2117 ; 220.0964 ; 163.0387$ & Glu-[lycibarbarspermidine B] ismoer & & $\checkmark$ & & \\
\hline B61 & 29.74 & $\mathrm{C}_{49} \mathrm{H}_{71} \mathrm{O}_{26} \mathrm{~N}_{3}$ & 1118.4481 & 1116.4180 & 1.45 & $956.3852 ; 794.3327 ; 632.3016 ; 470.2233 ; 220.0965 ; \mathbf{1 6 3 . 0 3 8 8}$ & 2Glu-[lycibarbarspermidine $\mathrm{O}$ ] isomer & & $\checkmark$ & & \\
\hline B62 & 29.88 & $\mathrm{C}_{35} \mathrm{H}_{49} \mathrm{O}_{15} \mathrm{~N}_{3}$ & 750.3069 & 748.2889 & 1.48 & $588.2748 ; 472.2429 ; 310.2118 ; 220.0965 ; 163.0389$ & Glu-[lycibarbarspermidine B] ismoer & & $\checkmark$ & & \\
\hline B63 & 30.03 & $\mathrm{C}_{25} \mathrm{H}_{33} \mathrm{O}_{6} \mathrm{~N}_{3}$ & 472.2389 & 470.2264 & 1.64 & $455.2168 ; 310.2119 ; 220.0965 ; 163.0388 ; 112.1121$ & $\begin{array}{l}N^{1} \text {-caffeoyl, } N^{3} \text {-dihydrocaffeoyl } \\
\text { spermidine }\end{array}$ & [34] & $\checkmark$ & $\checkmark$ & $\checkmark$ \\
\hline
\end{tabular}


Table 1. Cont.

\begin{tabular}{|c|c|c|c|c|c|c|c|c|c|c|c|}
\hline No & $\mathrm{RT}^{1}$ & Formula & {$[\mathrm{M}+\mathrm{H}]^{+}$} & {$[\mathrm{M}-\mathrm{H}]^{-}$} & ppm & MS/MS Fragments ${ }^{2}$ & Identification & Ref. $^{3}$ & $\mathrm{~F}^{4}$ & $\mathrm{~L}^{5}$ & $R^{6}$ \\
\hline B64 & 30.13 & $\mathrm{C}_{31} \mathrm{H}_{41} \mathrm{O}_{11} \mathrm{~N}_{3}$ & 632.2804 & 630.2628 & 1.57 & $470.2497 ; 382.1489 ; 308.1962 ; 220.0965 ; \mathbf{1 6 3 . 0 3 8 8}$ & lycibarbarspermidine $\mathrm{N}$ isomer & & $\checkmark$ & & \\
\hline B65 & 30.14 & $\mathrm{C}_{28} \mathrm{H}_{41} \mathrm{O}_{8} \mathrm{~N}_{3}$ & 548.3005 & & 7.11 & 530.2393; 474.2629; 293.1877; 222.1940; 165.0555; 128.1080 & Dihydrocaffeoyl spermidine derivative & & & & $\checkmark$ \\
\hline B66 & 30.14 & $\mathrm{C}_{34} \mathrm{H}_{28} \mathrm{O}_{12} \mathrm{~N}_{4}$ & 705.3398 & & 7.98 & $\begin{array}{c}\text { 687.3212; 531.3211; 467.2042; 310.2160; 293.1826; } \\
\text { 222.1139; } 165.0559\end{array}$ & Dihydrocaffeoyl spermine derivative & & & & $\checkmark$ \\
\hline B67 & 30.14 & $\mathrm{C}_{37} \mathrm{H}_{51} \mathrm{O}_{16} \mathrm{~N}_{3}$ & 794.3336 & 792.3145 & 0.76 & $632.2804 ; 470.2540 ; 308.1963 ; 220.0965 ; 163.0388$ & [lycibarbarspermidine $\mathrm{O}$ ] isomer & & $\checkmark$ & & \\
\hline B68 & 30.19 & $\mathrm{C}_{30} \mathrm{H}_{46} \mathrm{O}_{6} \mathrm{~N}_{4}$ & 559.3531 & & 1.89 & $395.2663 ; 307.2033 ; 236.1295 ; 222.123 ; 165.0561$ & Dihydrocaffeoyl spermine derivative & & & & $\checkmark$ \\
\hline B69 & 30.19 & $\mathrm{C}_{28} \mathrm{H}_{40} \mathrm{O}_{7} \mathrm{~N}_{3}$ & 530.2891 & 528.2696 & 1.68 & $474.2629 ; 310.2142 ; 293.1877 ; 222.1140 ; 165.0558$ & Propionyl-dihydrocaffeoyl spermidine & & & & $\checkmark$ \\
\hline B70 & 30.56 & $\mathrm{C}_{25} \mathrm{H}_{31} \mathrm{O}_{6} \mathrm{~N}_{3}$ & 470.2278 & 468.2115 & 1.54 & $308.1962 ; 291.1698 ; 234.1121 ; 220.0965 ; 163.0388$ & $N, N^{\prime}$-dicaffeoylspermidine & [33] & $\checkmark$ & $\checkmark$ & \\
\hline B71 & 30.82 & $\mathrm{C}_{34} \mathrm{H}_{46} \mathrm{O}_{11} \mathrm{~N}_{4}$ & 687.3308 & 685.3051 & 7.59 & $670.3035 ; 523.2778 ; 449.1942 ; 293.1878 ; 222.1139$ & Dihydrocaffeoyl spermine derivative & & & & $\checkmark$ \\
\hline B72 & 31.45 & $\mathrm{C}_{37} \mathrm{H}_{50} \mathrm{O}_{9} \mathrm{~N}_{4}$ & 695.3701 & 693.3464 & 1.28 & $678.3438 ; 531.3215 ; 457.2365 ; 293.1879 ; 222.1140 ; 165.0559$ & $N^{1}, N^{4}, N^{12}$-tris(dihydrocaffeoyl)spermine & [35] & & & $\checkmark$ \\
\hline B73 & 30.71 & $\mathrm{C}_{46} \mathrm{H}_{46} \mathrm{O}_{18} \mathrm{~N}_{4}$ & 963.4437 & 961.4240 & 0.87 & $\begin{array}{c}\text { 945.4429; 621.3273; } 455.2382 ; 384.1644 ; 293.1882 ; \\
\text { 222.1121; } 165.0557\end{array}$ & Ddihydrocaffeoyl spermine derivative & & $\checkmark$ & & \\
\hline B74 & 31.79 & $\mathrm{C}_{30} \mathrm{H}_{45} \mathrm{O}_{8} \mathrm{~N}_{3}$ & 576.3314 & 574.3247 & 1.45 & 544.3050; 512.2786; 412.2842; 293.1877; 222.1139; 165.0558 & Ddihydrocaffeoyl spermidine derivative & & & & $\checkmark$ \\
\hline $\mathrm{C} 1$ & 8.30 & $\mathrm{C}_{13} \mathrm{H}_{18} \mathrm{O}_{3} \mathrm{~N}_{2}$ & 251.1409 & 249.1227 & 1.41 & 234.0990; 163.0697; 144.0616; 126.0558; 115.0591 & $N$-caffeoylpu & [36] & $\checkmark$ & $\checkmark$ & $\checkmark$ \\
\hline C2 & 10.26 & $\mathrm{C}_{13} \mathrm{H}_{18} \mathrm{O}_{3} \mathrm{~N}_{2}$ & 251.1410 & & 1.69 & $234.1142 ; \mathbf{1 6 3 . 0 4 0 2} ; 145.0296 ; 115.0876$ & $\mathrm{~N}$-caffeoylputrescine isomer & [36] & $\checkmark$ & $\checkmark$ & \\
\hline $\mathrm{C} 3$ & 16.49 & $\mathrm{C}_{15} \mathrm{H}_{22} \mathrm{O}_{4} \mathrm{~N}_{2}$ & 295.1674 & & 1.20 & $278.1412 ; 207.0670 ; 175.0409 ; 147.0453 ; 129.1394$ & coumaroyl amide derivative & [37] & & & $\checkmark$ \\
\hline $\mathrm{C} 4$ & 23.48 & $\mathrm{C}_{15} \mathrm{H}_{22} \mathrm{O}_{4} \mathrm{~N}_{2}$ & 295.1673 & & 1.17 & $278.1405 ; 222.1142 ; 207.0666 ; 175.0412 ; 147.0451$ & coumaroyl amide derivative & [37] & & & $\checkmark$ \\
\hline C5 & 30.22 & $\mathrm{C}_{21} \mathrm{H}_{29} \mathrm{O}_{9} \mathrm{~N}$ & 476.1905 & 474.2481 & 2.14 & $314.1381 ; 222.1119 ; 177.0545 ; 145.0284 ; 121.0648$ & $\begin{array}{c}\mathrm{N} \text {-feruloyl-3-O-glucopyranosyl-tyramine } \\
\text { ismoer }\end{array}$ & [38] & $\checkmark$ & & \\
\hline C6 & 30.42 & $\mathrm{C}_{32} \mathrm{H}_{45} \mathrm{O}_{11} \mathrm{~N}_{3}$ & 648.3118 & 646.2941 & 1.32 & $486.2590 ; 310.2119 ; 234.1$ & feruloyl-tyramine derivatives & & $\checkmark$ & & \\
\hline $\mathrm{C} 7$ & 31.30 & & 302.1 & 3 & 1.46 & $286.0265 ; 245.8081 ; \mathbf{1 6 5 . 0}$ & A4 (caffeoyl-tyramine derivatives) & [32] & & & $\checkmark$ \\
\hline $\mathrm{C} 8$ & 31.34 & $\mathrm{C}_{14} \mathrm{H}_{14} \mathrm{O}_{5} \mathrm{~N}_{2}$ & 486.2587 & 484.2422 & 1.07 & 469.2316; 310.2118; 234.1120; $177.0544 ; 145.0283 ; 121.0649$ & feruloyl-tyramine derivatives & & $\checkmark$ & & \\
\hline C9 & 31.83 & $\mathrm{C}_{21} \mathrm{H}_{29} \mathrm{O}_{9} \mathrm{~N}$ & 476.1905 & 474.2481 & 2.07 & $314.1381 ; 222.1118 ; 177.0545 ; 145.0284 ; 121.0648$ & $\mathrm{~N}$-feruloyl-4-O-glucopyranosyl-tyramine & [38] & $\checkmark$ & & \\
\hline $\mathrm{C} 10$ & 32.09 & $\mathrm{C}_{36} \mathrm{H}_{36} \mathrm{O}_{8} \mathrm{~N}_{2}$ & 625.2537 & 623.1578 & 1.73 & $\begin{array}{c}\text { 462.1902; 351.0856; 293.08033; 201.0544; 175.0402; } \\
\text { 149.0609; } 121.0648\end{array}$ & Lyciumamide A & [39] & $\checkmark$ & & \\
\hline C11 & 32.22 & $\mathrm{C}_{36} \mathrm{H}_{49} \mathrm{O}_{16} \mathrm{~N}_{3}$ & 498.2592 & 498.2576 & 1.41 & $480.2489 ; 322.2119 ; 234.1126 ; 177.0545 ; 145.0284$ & feruloyl-tyramine derivatives & & $\checkmark$ & & \\
\hline $\mathrm{C} 12$ & 32.57 & & 300.1256 & 298.1 & 0.4 & $253.8823 ; 163.0402 ; 121.0658$ & A5 (caffeoyl-ty & [32] & & & $\checkmark$ \\
\hline $\mathrm{C} 13$ & 32.98 & $\mathrm{C}_{17} \mathrm{H}_{17} \mathrm{O}_{3} \mathrm{~N}$ & 284.1275 & 282.1116 & 2.29 & $261.0436 ; 164.0705 ; \mathbf{1 4 7 . 0 4 3 9} ; 121.0648$ & $N$-p-cis-Coumaroyl tyramine & [40] & $\checkmark$ & $\checkmark$ & \\
\hline $\mathrm{C} 14$ & 33.33 & $\mathrm{C}_{18} \mathrm{H}_{19} \mathrm{O}_{4} \mathrm{~N}$ & 314.1382 & 312.1221 & 1.67 & $\begin{array}{c}274.8365 ; 243.1029 ; 220.0979 ; 195.0847 ; \mathbf{1 7 7 . 0 5 5 1} ; \\
145.0289 \cdot 121.0653\end{array}$ & N-cis-feruloyl-tyramine & [8] & $\checkmark$ & & $\checkmark$ \\
\hline C15 & 33.47 & $\mathrm{C}_{17} \mathrm{H}_{17} \mathrm{O}_{3} \mathrm{~N}$ & 284.1275 & 2.1116 & 2.29 & $261.0436 ; 164.0705 ; \mathbf{1 4 7 . 0 4 3 9} ; 121.0648$ & N-p-trans-Coumaroyl tyramine* & & $\checkmark$ & $\checkmark$ & \\
\hline C16 & 33.62 & & 5 & 14 & 1.36 & $462.1963 ; 325.1065 ; 210.0545 ; 177.0546 ; 121.0648$ & & [36] & $\checkmark$ & & \\
\hline C17 & 33.81 & $\mathrm{O}_{4} \mathrm{~N}$ & 314.1382 & 312.1221 & 1.51 & $244.0981 ; 220.0979 ; 194.0822 ; 177.0555 ; 145.0292 ; 121.0653$ & $N$-trans-feruloyl-tyramine & [8] & $\checkmark$ & $\checkmark$ & $\checkmark$ \\
\hline C18 & 34.02 & $\mathrm{C}_{19} \mathrm{H}_{19} \mathrm{O}_{5} \mathrm{~N}$ & 344.1520 & 1323 & 1.25 & $282.3545 ; \mathbf{1 7 7 . 0 5 5 9 ;} 145.0295$ & A12 (feruloyl-tyramine derivatives) & [32] & & & $\checkmark$ \\
\hline C19 & 34.98 & & 643.2 & 年 & 1.89 & $462.1903 ; 325.1063 ; 201.05$ & feruloyl-tyramine derivatives & & $\checkmark$ & & \\
\hline $\mathrm{C} 20$ & 35.07 & $\mathrm{C}_{28} \mathrm{H}_{29} \mathrm{O}_{7} \mathrm{~N}$ & 492.2010 & 490.1842 & 1.46 & $462.1909 ; 325.1066 ; 293.0805 ; 201.0546 ; 175.0769 ; 121.0649$ & Lyciumamide $\mathrm{C}$ & [39] & $\checkmark$ & & \\
\hline $\mathrm{C} 21$ & 36.57 & $\mathrm{C}_{54} \mathrm{H}_{53} \mathrm{O}_{12} \mathrm{~N}_{3}$ & 936.3691 & & 1.20 & $\begin{array}{c}\text { 771.2914; } 634.20612 ; \mathbf{4 7 1 . 1 4 2 9} ; 375.0859 ; 263.0896 ; \\
\text { 203.5736; } 121.0659\end{array}$ & melongenamide $\mathrm{D}$ isomer & [41] & $\checkmark$ & & \\
\hline D1 & 24.74 & $\mathrm{C}_{33} \mathrm{H}_{40} \mathrm{O}_{2} 1$ & 773.2195 & 771.1937 & 1.21 & 611.140; 464.0762; 303.0325; 163.0399 & Quercetin-3-O-Glu-7-O-Rha isomer & [42] & & $\checkmark$ & \\
\hline D2 & 26.89 & $\mathrm{C}_{27} \mathrm{H}_{30} \mathrm{O}_{17}$ & 627.1610 & 625.1376 & 1.66 & $\begin{array}{l}585.6912 ; 303.0520 ; 285.0409 ; 257.043 ; 243.5646 ; \\
201.4349: 12902379\end{array}$ & Quercetin-3,7-O-diGlu & [42] & $\checkmark$ & $\checkmark$ & \\
\hline D3 & 26.98 & $\mathrm{C}_{33} \mathrm{H}_{40} \mathrm{O}_{21}$ & 773.2195 & 771.1940 & 1.84 & $726.3508 ; 559.7092 ; 465.1061 ; 303.0521 ; 228.4964 ; 129.0548$ & Quercetin-3-O-Soph-7-O-Rha & [42] & & $\checkmark$ & \\
\hline
\end{tabular}


Table 1. Cont.

\begin{tabular}{|c|c|c|c|c|c|c|c|c|c|c|c|}
\hline No & RT $^{1}$ & Formula & {$[\mathrm{M}+\mathrm{H}]^{+}$} & {$[\mathrm{M}-\mathrm{H}]^{-}$} & ppm & MS/MS Fragments ${ }^{2}$ & Identification & Ref. $^{3}$ & $\mathrm{~F}^{4}$ & $\mathrm{~L}^{5}$ & $\mathbf{R}^{6}$ \\
\hline $\mathrm{D} 4$ & 27.74 & $\mathrm{C}_{33} \mathrm{H}_{40} \mathrm{O}_{21}$ & 773.2127 & 771.1940 & 1.08 & 611.3322; 472.2473; 303.0492; 220.0965; 163.0399; 129.0541 & Quercetin-3-O-Rut-7-O-Glu & [42] & $\checkmark$ & & $\checkmark$ \\
\hline D5 & 31.39 & $\mathrm{C}_{27} \mathrm{H}_{30} \mathrm{O}_{16}$ & 611.1600 & 609.1417 & 1.03 & $\begin{array}{c}\text { 449.1113; 465.1061; 303.0492; 285.0413; 257.046; } \\
\text { 201.0561129.05449 }\end{array}$ & Rutin * & & $\checkmark$ & $\checkmark$ & \\
\hline D6 & 31.98 & $\mathrm{C}_{27} \mathrm{H}_{30} \mathrm{O}_{15}$ & 595.1703 & 593.1472 & 1.63 & $\begin{array}{c}\text { 465.5521; 329.0679; } 287.0529 ; 258.2196 ; 243.5895 ; \\
\text { 230.3383; } 129.0553\end{array}$ & Kaempferol-3-O-Glu-7-O-Rha & [42] & $\checkmark$ & $\checkmark$ & \\
\hline E1 & 33.67 & $\mathrm{C}_{45} \mathrm{H}_{72} \mathrm{O}_{17}$ & 885.4835 & & 0.87 & $\begin{array}{c}\text { 867.4724; 415.3229; } 299.2362 ; 271.2052 ; 253.1947 ; \\
\text { 215.1792; } 157.1011\end{array}$ & Gracillin & [43] & $\checkmark$ & & \\
\hline E2 & 33.72 & $\mathrm{C}_{45} \mathrm{H}_{73} \mathrm{O}_{16} \mathrm{~N}$ & 884.5084 & 882.4800 & 1.13 & $\begin{array}{c}\text { 866.4896; 720.4325; 576.3898; } 414.3403 ; 396.3259 ; \\
271.2054 ; 253.1949\end{array}$ & Solasonine* & & $\checkmark$ & & \\
\hline E3 & 33.77 & $\mathrm{C}_{51} \mathrm{H}_{84} \mathrm{O}_{22}$ & 1049.5518 & & 0.90 & 887.4990; 743.3851; 417.3355; 273.2207; 255.2107 & parillin & [44] & $\checkmark$ & & \\
\hline E4 & 33.87 & $\mathrm{C}_{45} \mathrm{H}_{75} \mathrm{O}_{16} \mathrm{~N}$ & 886.5152 & 884.4957 & 0.75 & $\begin{array}{r}\text { 868.5034; } 722.4475 ; 416.356 ; 398.3409 ; 273.2205 ; \\
255.2102 ; 173.1323\end{array}$ & 5,6-dihydrosolasonine * & & $\checkmark$ & & \\
\hline E5 & 33.92 & $\mathrm{C}_{45} \mathrm{H}_{73} \mathrm{O}_{18}$ & 903.5006 & & 1.21 & 741.4469; 597.3309; 417.3389; 273.2230; 255.2124; 145.0506 & Timosaponin BIII & [45] & $\checkmark$ & & \\
\hline E6 & 34.49 & $\mathrm{C}_{47} \mathrm{H}_{77} \mathrm{O}_{17} \mathrm{~N}$ & 928.5253 & & 1.25 & $458.3620 ; 273.2207 ; 255.2102 ; 161.1323$ & Lycioside B & [1] & $\checkmark$ & & \\
\hline F1 & 3.93 & $\mathrm{C}_{9} \mathrm{H}_{11} \mathrm{O}_{2} \mathrm{~N}$ & 166.0867 & & 1.10 & $153.0416 ; 142.9681 ; \mathbf{1 2 0 . 0 8 1 4} ; 103.0548$ & Phenylalanine isomer & [1] & $\checkmark$ & $\checkmark$ & $\checkmark$ \\
\hline F2 & 5.51 & $\mathrm{C}_{9} \mathrm{H}_{11} \mathrm{O}_{3} \mathrm{~N}$ & 166.0877 & & 1.13 & $142.9681 ; 138.0554 ; \mathbf{1 2 0 . 0 8 1 2} ; 103.0548$ & Phenylalanine isomer & & $\checkmark$ & $\checkmark$ & $\checkmark$ \\
\hline F3 & 9.95 & $\mathrm{C}_{11} \mathrm{H}_{9} \mathrm{O}_{2} \mathrm{~N}$ & 188.0721 & & 1.95 & $170.0613 ; \mathbf{1 4 6 . 0 4 1 2} ; 118.0611$ & 3-amino-2-naphthoic acid & [46] & $\checkmark$ & $\checkmark$ & $\checkmark$ \\
\hline $\mathrm{F} 4$ & 10.44 & & & 371.0991 & 0.11 & $205.0506 ; 163.0398 ; 145.9288 ; 119.0498$ & & & $\checkmark$ & & \\
\hline F5 & 31.16 & & & 568.3110 & & $539.2707 ; 363.2378 ; 268.0598 ; 135.0436$ & & & & & $\checkmark$ \\
\hline F6 & 31.29 & & 748.3361 & & & $\begin{array}{c}\text { 609.3981; 399.1691; 360.1675; 314.1518; } 215.0829 ; \\
\text { 171.0929; } 136.0770\end{array}$ & & & & & $\checkmark$ \\
\hline F7 & 31.69 & & & 630.3469 & & 498.3042; 469.2647; $387.2245 ; 241.0723 ; 151.2698$ & & & & & $\checkmark$ \\
\hline F8 & 32.56 & & & 298.1066 & & $256.0959 ; 178.0492 ; \mathbf{1 3 5 . 0 4 3 5}$ & & & & & $\checkmark$ \\
\hline F9 & 32.91 & & & 647.3257 & & 618.1393; 483.2572; 412.2080; 395.2055; 161.4154 & & & & & $\checkmark$ \\
\hline F10 & 34.22 & & 897.3956 & 895.3695 & & $\begin{array}{c}\text { 879.3847; 689.3096; 486.2017; 468.1911; 422.583; } \\
\text { 395.1742; } 159.0929\end{array}$ & & & & & $\checkmark$ \\
\hline F11 & 34.23 & & & 898.4049 & & 690.3203; 527.2580; 387.1645; 203.0810; 153.0655 & & & & & $\checkmark$ \\
\hline
\end{tabular}

(show the fragmentation ions in negative ionization mode); the number in bold means the most abundant product ion. ${ }^{3}$ Ref. represents the references; ${ }^{4-6} \mathrm{~F}, \mathrm{~L}$, R represent fruits, leaves and Root barks respectively. * Represents the compounds confirmed by standards 
Compounds B18, B31, B42, B48 and B55 displaying $[\mathrm{M}+\mathrm{H}]^{+}$ions at $m / z 634.2959\left(\mathrm{C}_{31} \mathrm{H}_{44} \mathrm{O}_{11} \mathrm{~N}_{3}\right.$, Cal. 634.2970, mass error $1.31 \mathrm{ppm}$ ) and fragment ions at $m / z$ 310, 220 and 163 were identified to be five positional isomers of diglycosyl-caffeoyl spermidine. Their common fragment ion at $\mathrm{m} / \mathrm{z} 472$ is formed by the cleavage of one glucosyl (162 Da, Figure S11). Zhou et al. reported 15 dicaffeoylspermidine derivatives in the fruit of L. barbarum [32]. Here, we further found more dicaffeoylspermidine and dicaffeoylspermine derivatives; for example, compounds B46, B50 and B54 found in fruit of L. barbarum showing similar $[\mathrm{M}+\mathrm{H}]^{+}$ions and fragment ions (Figure S12) were tentatively identified to be isomers of dicaffeoylspermidine derivative with four glucosyls. Compound B46 readily yielded a strong $[\mathrm{M}+\mathrm{H}]^{+}$ion at $m / z 1120.4628\left(\mathrm{C}_{49} \mathrm{H}_{74} \mathrm{O}_{26} \mathrm{~N}_{3}\right.$, Cal. 1120.4555 , mass error $\left.1.58 \mathrm{ppm}\right)$ and main fragment ions at $m / z 958.4113\left[\mathrm{M}+\mathrm{H}-\mathrm{C}_{6} \mathrm{H}_{10} \mathrm{O}_{5}\right]^{+}, m / z 796.3885\left[\mathrm{M}+\mathrm{H}-\mathrm{C}_{6} \mathrm{H}_{10} \mathrm{O}_{5}-\mathrm{C}_{6} \mathrm{H}_{10} \mathrm{O}_{5}\right]^{+}, m / z 634.2957$ $\left[\mathrm{M}+\mathrm{H}-\mathrm{C}_{6} \mathrm{H}_{10} \mathrm{O}_{5}-\mathrm{C}_{6} \mathrm{H}_{10} \mathrm{O}_{5}-\mathrm{C}_{6} \mathrm{H}_{10} \mathrm{O}_{5}\right]^{+}, m / z$ 472.2471 $\left[\mathrm{M}+\mathrm{H}-\mathrm{C}_{6} \mathrm{H}_{10} \mathrm{O}_{5}-\mathrm{C}_{6} \mathrm{H}_{10} \mathrm{O}_{5}-\mathrm{C}_{6} \mathrm{H}_{10} \mathrm{O}_{5}\right.$ $\left.-\mathrm{C}_{6} \mathrm{H}_{10} \mathrm{O}_{5}\right]^{+}$, corresponding to successive losses of glucosyl units. Similarly, compound B61 that yielded a strong $[\mathrm{M}+\mathrm{H}]^{+}$ion at $m / z 1118.4481\left(\mathrm{C}_{49} \mathrm{H}_{72} \mathrm{O}_{26} \mathrm{~N}_{3}\right.$, Cal. 1118.4396, mass error $\left.1.342 \mathrm{ppm}\right)$ also contains four hexoses (Figure S13).

The detected phenolic amides mainly consist of feruloyl, caffeoyl or coumaroyl groups and different amino groups; they generated fragment ions at $m / z 177,163$ and 147, respectively. Many of them contained a tyramine moiety $(137 \mathrm{Da})$, which lost a $\mathrm{NH}_{3}$ to generate a vinylphenol ion at $\mathrm{m} / \mathrm{z}$ 121. Compound $\mathrm{C} 15$ displaying a $[\mathrm{M}+\mathrm{H}]^{+}$at $m / z 284.1274\left(\mathrm{C}_{17} \mathrm{H}_{18} \mathrm{O}_{3} \mathrm{~N}\right.$, Cal. 284.1281, mass error $1.29 \mathrm{ppm}$ ) was identified to be $N$-p-trans-coumaroyltyramine and confirmed by a standard, which generated fragment ions at $m / z 147$ and 121 (Figure S6). The ion at $m / z 147.0445$ was formed by the neutral loss of a tyramine unit. Compound C15 displaying a $[\mathrm{M}+\mathrm{H}]^{+}$at $m / z 314.1382\left(\mathrm{C}_{18} \mathrm{H}_{20} \mathrm{O}_{4} \mathrm{~N}\right.$, Cal. 314.1387, mass error $1.67 \mathrm{ppm}$ ) was identified to be $N$-feruloyltyramine; the key product ions at $\mathrm{m} / \mathrm{z} 177.0550$ and 121.0658 indicate the existence of feruloyl and tyramine moieties (Figure S14).

Flavones included six compounds containing quercetin and kaempferol groups, respectively. These groups generated characteristic ions at $m / z 303$ (quercetin), 287 (kaempferol) and a series of fragment ions that continuously lost a $\mathrm{CO}(28 \mathrm{Da})$ or $\mathrm{CO}_{2}(44 \mathrm{Da})$. Compound D5 displaying a $[\mathrm{M}+\mathrm{H}]^{+}$ ion at $m / z 611.1600\left(\mathrm{C}_{27} \mathrm{H}_{31} \mathrm{O}_{16}\right.$, Cal. 611.1607, mass error $\left.1.03 \mathrm{ppm}\right)$ and fragment ions at $m / z$ 303.0492, 285.0413, 257.046, 201.0561 was identified to be rutin and confirmed by a rutin standard (Figure S7). The fragment ions at $m / z 465.1061\left[\mathrm{M}+\mathrm{H}-\mathrm{C}_{6} \mathrm{H}_{10} \mathrm{O}_{4}\right]^{+}, 303.0492\left[\mathrm{M}+\mathrm{H}-\mathrm{C}_{6} \mathrm{H}_{10} \mathrm{O}_{4}-\mathrm{C}_{6} \mathrm{H}_{10} \mathrm{O}_{5}\right]^{+}$, $285.0413\left[\mathrm{M}+\mathrm{H}-\mathrm{C}_{6} \mathrm{H}_{10} \mathrm{O}_{4}-\mathrm{C}_{6} \mathrm{H}_{10} \mathrm{O}_{5}-\mathrm{H}_{2} \mathrm{O}\right]^{+}$, correspond to successive losses of rhamnosyl, glucosyl and $\mathrm{H}_{2} \mathrm{O}$. The fragment ion at $\mathrm{m} / z 201.05612$ was formed by the successive loss of three $\mathrm{CO}$ units from the ion at $m / z$ 285.0413. In the same manner, compound D6 displaying a $[\mathrm{M}+\mathrm{H}]^{+}$ion at $m / z 595.1703\left(\mathrm{C}_{27} \mathrm{H}_{31} \mathrm{O}_{15}\right.$, Cal. 595.1657, mass error $\left.0.59 \mathrm{ppm}\right)$ and key product ions at $m / z 287.0529$, 258.2196, 243.5895 and 230.3383 was identified to be kaempferol-O-Glu-O-Rha. The fragment ions at $m / z 449.1408\left[\mathrm{M}+\mathrm{H}-\mathrm{C}_{6} \mathrm{H}_{10} \mathrm{O}_{4}\right]^{+}, 287.0528\left[\mathrm{M}+\mathrm{H}-\mathrm{C}_{6} \mathrm{H}_{10} \mathrm{O}_{4}-\mathrm{C}_{6} \mathrm{H}_{10} \mathrm{O}_{5}\right]^{+}$, correspond to the loss of the rhamnosyl and glucosyl units (Figure S15).

Saponins contain a steroid skeleton and generate key fragment ions at $m / z 273 / 271$ and 255/253 depending on the presence of a single bond or a double bond at the positions 5 and 6 (Figure 1 and Figure S2). Compounds E2 and E4 were identified to be solasonine and 5,6-dihydrosolasonine by the standards and fragment ions as described above. Compound E3 was tentatively identified to be parillin with $[\mathrm{M}+\mathrm{H}]^{+}$at $m / z 1049.5527\left(\mathrm{C}_{45} \mathrm{H}_{76} \mathrm{O}_{16} \mathrm{~N}\right.$, Cal. 1049.5527, mass error $\left.0.90 \mathrm{ppm}\right)$, and fragment ions at $m / z 887.4990\left[\mathrm{M}+\mathrm{H}-\mathrm{C}_{6} \mathrm{H}_{10} \mathrm{O}_{5}\right]^{+}, 743.3850\left[\mathrm{M}+\mathrm{H}-\mathrm{C}_{6} \mathrm{H}_{10} \mathrm{O}_{5}-\mathrm{C}_{6} \mathrm{H}_{10} \mathrm{O}_{4}\right]^{+}, 579.3932[\mathrm{M}+\mathrm{H}-$ $\left.\mathrm{C}_{6} \mathrm{H}_{10} \mathrm{O}_{5}-\mathrm{C}_{6} \mathrm{H}_{10} \mathrm{O}_{4}-\mathrm{C}_{6} \mathrm{H}_{10} \mathrm{O}_{4}\right]^{+}, 417.3392\left[\mathrm{M}+\mathrm{H}-\mathrm{C}_{6} \mathrm{H}_{10} \mathrm{O}_{5}-\mathrm{C}_{6} \mathrm{H}_{10} \mathrm{O}_{4}-\mathrm{C}_{6} \mathrm{H}_{10} \mathrm{O}_{4}-\mathrm{C}_{6} \mathrm{H}_{10} \mathrm{O}_{5}\right]^{+}$, 273.2232 and 255.2125 (Figure S16). It is worth mentioning that four of the six identified saponins, E1 (gracillin), E2 (solasonine), E4 (5,6-dihydrosolasonine) and E6 (lycioside B) have the same sugar moiety, solatriose, but other steroidal glycoalkaloids from Solanum plants consist of the same aglycones and different sugar moieties, such as solamargine (chacotriose + solasodine) and soladulcine A/B (chacotriose/lycotetraose + soladulcidine), which were not detected in the three parts of L. barbarum, suggesting that solatriose might be a characteristic sugar unit in L. barbarum. Saponins are rarely 
reported in L. barbarum [1,47]. All the saponins (E1-E6) reported here were found in L. barbarum for the first time.

Eleven other compounds were also detected, but their diagnostic ions were not included in the characteristic ion database. Consequently, these compounds were not classified.

In summary, a total of 131 compounds including 13 phenylpropanoids, 74 dicaffeoylspermidine or dicaffeoylspermine derivatives, 21 phenolic amides, six flavonoids, six saponins and 11 others were detected. Among them, 98 compounds were found in fruits, 28 compounds were found in leaves and 35 compounds were found in root barks of L. barbarum, suggesting that the fruits extract contains many more components than root barks and leaves ones. As shown in the total ion chromatograms of UPLC-HR-MS (Figure 3), the distribution of these constituents in the extracts of fruits, root barks and leaves are obviously different. A lot of dicaffeoylspermidine or dicaffeoylspermine derivatives were detected in fruits, leaves and root barks. Among them, kukoamine A (B7) and kukoamine B (B9) (dicaffeoylspermine isomers, main peaks around $20 \mathrm{~min}$ in positive/negative modes) and propionyl-dicaffeoylspermidine (B69, main peak around $30 \mathrm{~min}$ in negative mode) were found in the extract of root barks in a very large amount, but not detected in leaves and fruits. Dicaffeoylspermidine isomers (B52, B63 and B70) were detected in a high amount in fruits and leaves, but were much lower in root barks. However, some glycosylated derivatives of kukoamine A/B isomers (such as B2, B3, $\mathrm{B} 4, \mathrm{~B} 6, \mathrm{~B} 8, \mathrm{~B} 66$ and B72) and many glycosylated derivatives of dicaffeoylspermidine (such as B13-20, B24-42, B30, B38, B44, B46-51, B53-62, B64 and B67) were mainly found in fruits, suggesting that dicaffeoylspermidines and dicaffeoylspermines were glycosylated in the fruit of L. barbarum. The difference of the distribution of dicaffeoylspermine and dicaffeoylspermidine derivatives suggests low chemical similarity between root barks and fruits/leaves. Based on Table 1, phenylpropanoids were mainly found in the fruits and leaves, but not detected in the root barks. Phenolic amides were found in all the three parts of L. barbarum plants. Flavonoids were mainly found in the fruits and leaves in a high amount, e.g., rutin (D5). Saponins were only detected in the fruits of L. barbarum.

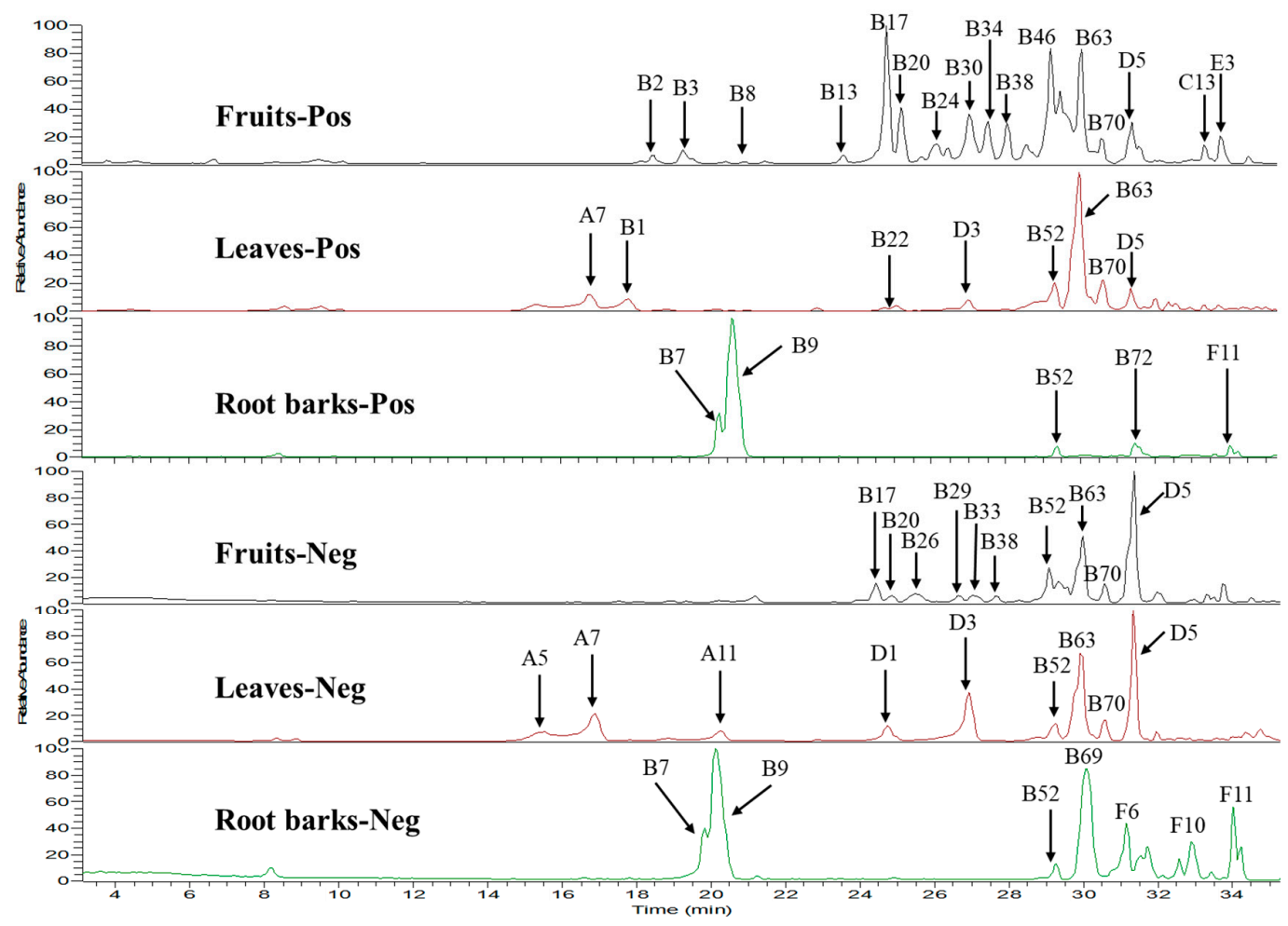

Figure 3. Total ion chromatograms (TICs) of extracts of fruits, leaves and root barks in positive and negative ion mode using UPLC-HR-MS. All the compound numbers were same with those shown in Table 1. 


\subsection{Quantitative Analysis of Seven Compounds in the Fruits, Leaves and Root Barks}

The quantitative analysis of seven compounds in the extracts was performed by UPLC-Qtrap-MS with MRM mode owing to its high sensitivity, specificity, and selectivity in the quantitation of trace compounds in complex matrices [18]. The method validation data are listed in Table S2. The calibration curves of the seven standards showed good linearity ( $\mathrm{r}$ from 0.989 to 0.998 ). The limit of detection (LOD) and the limit of quantification (LOQ) for each standard were $0.08-0.26 \mu \mathrm{g} / \mathrm{mL}$ and $0.02-0.07 \mu \mathrm{g} / \mathrm{mL}$. Both intra-day $(n=3)$ and inter-day $(n=6)$ precision was evaluated and the RSDs of the seven standards were less than $4.57 \%$ and $3.86 \%$. The recoveries obtained in this study were in the range of $92-112 \%$ (Table S3) with low RSDs ( $<7 \%$ ) of all standards, demonstrating that the analytical method developed has high accuracy and good reproducibility.

The quantitative results were shown in Table 2. Kukoamine B was only detected in the root barks in a very high amount $(10.9 \mathrm{mg} / \mathrm{g}$ dry powder). The amount of rutin (D5) in leaves (663.45 $\mu \mathrm{g} / \mathrm{g}$ dry leaves) was much higher than that in fruits ( $93 \mu \mathrm{g} / \mathrm{g}$ dry fruits). However, it was not found in root barks, which was consistent with the results of the above qualitative assay (Figure 3). Chlorogenic acid was only observed in leaves in a high amount (1577 $\mu \mathrm{g} / \mathrm{g}$ dry leaves). $N$ - $p$-trans-coumaroyltyramine was found in both fruits and leaves in a little amount $(<15 \mu \mathrm{g} / \mathrm{g}$ dry powder). Dihydrosolasonine was only found in fruits in an amount of $43 \mu \mathrm{g} / \mathrm{g}$ dry fruit. The major steroidal glycoalkaloid in Solanaceae, solasonine, was only found in fruits in a very low amount $(2.16 \mu \mathrm{g} / \mathrm{g}$ dry fruits), which is much lower than that found in Solanum xanthocarpum $(800 \mu \mathrm{g} / \mathrm{g})$ [48].

Table 2. The amounts of seven standards in $1 \mathrm{~g}$ dry fruits, leaves and root barks.

\begin{tabular}{cccc}
\hline Compounds & Fruits $(\mu \mathrm{g} / \mathrm{g})$ & Leaves $(\mu \mathrm{g} / \mathrm{g})$ & Root Barks $(\mu \mathrm{g} / \mathrm{g})$ \\
\hline Kukoamine B & - & - & $10,900 \pm 3$ \\
Scopolin & $12.7 \pm 0.08$ & - & - \\
Chlorogenic acid & - & $1577 \pm 4$ & - \\
Rutin & $93 \pm 5$ & $663 \pm 15$ & - \\
Solasonine & $2.16 \pm 0.02$ & - & - \\
5,6-Dihydrosolasonine & $43 \pm 3$ & - & - \\
N-p-trans-Coumaroyltyramine & $3.33 \pm 0.02$ & $14 \pm 1$ & - \\
\hline
\end{tabular}

Results are expressed as means $\pm \mathrm{SD}, n=3$.

\subsection{Antioxidative Activity Assays}

Since a lot of phenolic compounds (phenylpropanoids, dicaffeoylspermidine/dicaffeoyl-spermine derivatives, phenolic amides, flavonoids) were found in the extracts, their antioxidative activities were compared using 2,2-diphenyl-1-picrylhydrazyl (DPPH), 2,2'-azinobis-(3-ethyl-benzthiazoline6-sulphonate) (ABTS) and ferric reducing ability of plasma (FRAP) assays [49,50]. The results demonstrated that all the extracts showed antioxidative activities (Table 3). Both DPPH and ABTS assays showed that root bark extract possessed the strongest free radical-scavenging capacity, leaf extract was the second, and fruit extract showed much lower free radical-scavenging capacity than either leaf or root bark extracts. The FRAP assay also showed that the fruit extract had the weakest reducing ability. The strong antioxidative activity of the root bark extract could be explained by the huge amount of kukoamine $\mathrm{A} / \mathrm{B}$ and propionyl-dicaffeoylspermidine in the root barks [51]. The higher amount of chlorogenic acid and rutin in leaves than those in fruits might explain the higher antioxidative activity of the leaf extract. The overall much higher antioxidant ability of leave and root bark extracts than that of the fruit extract is different from our common understanding that the fruits have strong antioxidant ability. 
Table 3. Antioxidant capacity of fruit, leaf and root bark extracts.

\begin{tabular}{|c|c|c|c|}
\hline Extracts & $\begin{array}{c}\mathrm{DPPH}\left(\mathrm{IC}_{50}\right)^{1} \\
(\mu \mathrm{g} / \mathrm{mL})\end{array}$ & $\begin{array}{c}\text { ABTS }\left(\mathrm{IC}_{50}\right)^{2} \\
(\mu \mathrm{g} / \mathrm{mL})\end{array}$ & $\begin{array}{c}\text { FRAP }\left(\text { RC }_{50}\right)^{3} \\
(\mu \mathrm{g} / \mathrm{mL})\end{array}$ \\
\hline Fruits & $1974.3 \pm 0.4$ & $247.0 \pm 0.8$ & $725 \pm 1.4$ \\
\hline Leaves & $123.5 \pm 0.5$ & $56 \pm 1$ & $192.6 \pm 0.02$ \\
\hline Root barks & $85.0 \pm 0.3$ & $40.7 \pm 0.4$ & $224 \pm 1$ \\
\hline Ascorbic acid 4 & $11.2 \pm 0.5$ & - & - \\
\hline Trolox 5 & - & $6 \pm 1$ & $37.7 \pm 0.3$ \\
\hline
\end{tabular}

${ }^{1} \mathrm{DPPH}\left(\mathrm{IC}_{50}\right)$ represents the extract concentration scavenging $50 \%$ of DPPH radical; ${ }^{2} \mathrm{ABTS}\left(\mathrm{IC}_{50}\right)$ represents the extract concentration scavenging $50 \%$ of $\mathrm{ABTS}$ radical; ${ }^{3} \mathrm{FRAP}\left(\mathrm{RC}_{50}\right)$ represents the extract concentration providing $50 \%$ reduction of $\mathrm{Fe}^{3+}$ to $\mathrm{Fe}^{2+} ;{ }^{4,5}$ represent the positive control; Results are expressed as means $\pm \mathrm{SD}, n=3$.

\subsection{Protective Effects of Extracts on $\mathrm{H}_{2} \mathrm{O}_{2}$-Induced Oxidative Stress in Cells}

$\mathrm{H}_{2} \mathrm{O}_{2}$ triggers oxidative damage through an increase of intracellular ROS. Therefore, the effects of extracts on the production of ROS in $\mathrm{H}_{2} \mathrm{O}_{2}$-exposed $\mathrm{L} 02$ cells were measured by $2^{\prime}, 7^{\prime}$-dichloro-dihydrofluorescein diacetate (DCFHDA) assay (Figure 4). ROS in cells can oxidize DCFH (no fluorescence) to form high fluorescent DCF. The results revealed that all the three extracts caused a dose-dependent attenuation of the $\mathrm{H}_{2} \mathrm{O}_{2}$-induced ROS production in $\mathrm{L} 02$ cells. The leaf and root bark extracts showed significantly higher antioxidative activity than that of fruit extract (Figure $4 \mathrm{a}-\mathrm{c}$ and Figure S17).

(a)

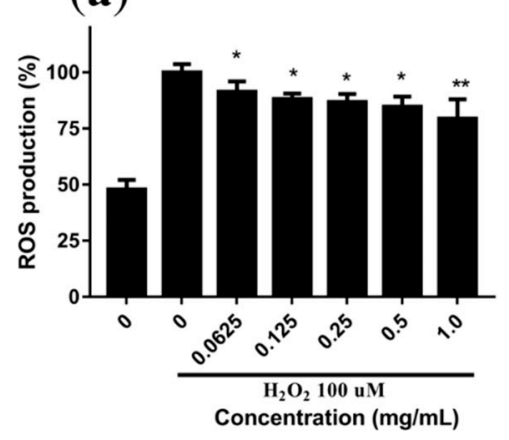

(d)
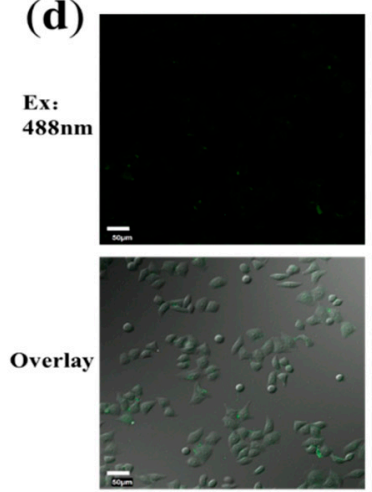

$\mathrm{H}_{2} \mathrm{O}_{2}$ Extracts (b)
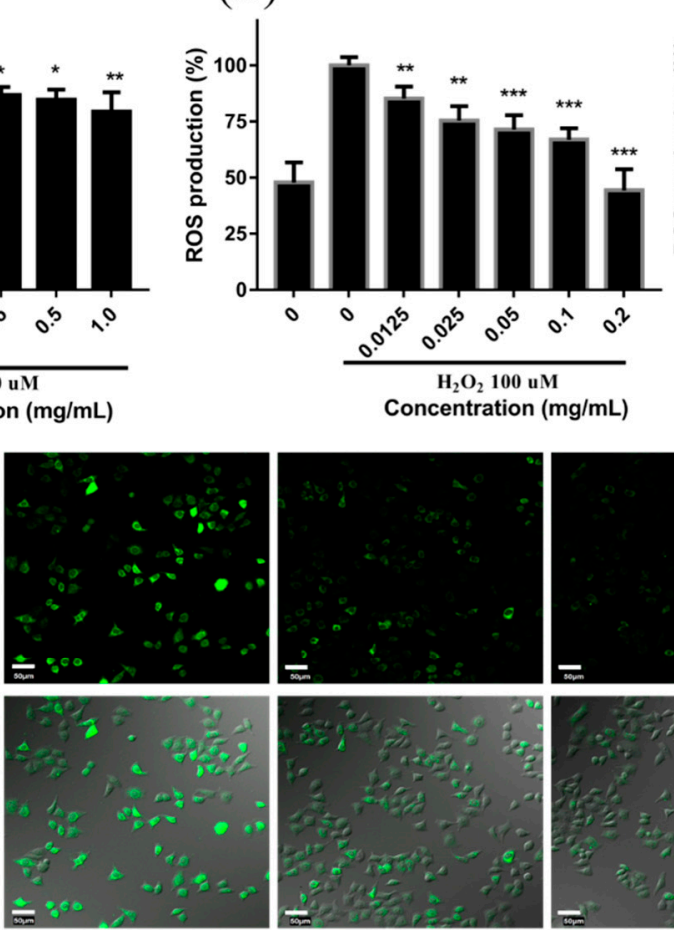

$+$ (c)
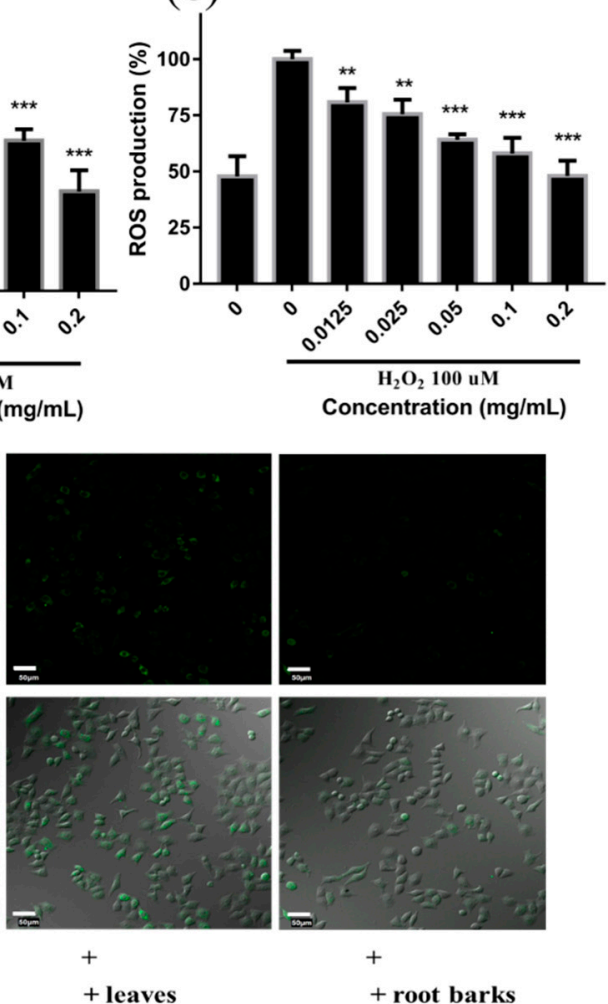

Figure 4. Protection effect of different concentrations of extracts from fruits (a), leaves (b) and root barks (c) on $100 \mu \mathrm{M} \mathrm{H}_{2} \mathrm{O}_{2}$-induced intracellular ROS production in L02 cells. Results were expressed as means $\pm \mathrm{SD}, n=3$. ${ }^{*} p<0.05,{ }^{* *} p<0.01$, ${ }^{* *} p<0.001$, vs. the $\mathrm{H}_{2} \mathrm{O}_{2}$ treated group. (d) Confocal imaging of intracellular ROS levels after $\mathrm{H}_{2} \mathrm{O}_{2}$ stimulation in the presence of extracts of fruits $(1 \mathrm{mg} / \mathrm{mL})$, leaves and root barks $(0.2 \mathrm{mg} / \mathrm{mL})$ (scale bar, $50 \mu \mathrm{m})$.

The ROS in cells treated by $0.25 \mathrm{mg} / \mathrm{mL}$ fruit extract was only reduced to $86 \%$, while the ROS in cells treated by $0.2 \mathrm{mg} / \mathrm{mL}$ leaf and root extracts were reduced to $44 \%$ and $48 \%$ respectively. The 
confocal imaging of cells (Figure 4d) also showed that the three extracts greatly reduced the fluorescence intensity in cells, and the fruit extract showed the weakest effect. This set of results was consistent with the results of the above antioxidative activity analysis in solutions.

\subsection{In Vitro Assays of Cytotoxicity}

Glycoalkaloids are reported to have anticarcinogenic activity because of their cytotoxicity $\left(\mathrm{IC}_{50}=50 \mu \mathrm{g} / \mathrm{mL}\right)$ [52]. Thus, the cytotoxicities of the fruit, leaf and root bark extracts to L02 cells were measured by a CCK-8 assay. L02 cell line was derived from the human hepatic and have been widely used in the evaluation of basic cytotoxicity profiles of drug candidates. As shown in Figure 5, the fruit extract did not show cytotoxicity at $1 \mathrm{mg} / \mathrm{mL}$ but showed cytotoxicity at $2 \mathrm{mg} / \mathrm{mL}$. The leaf extract showed weak cytotoxicity at $1 \mathrm{mg} / \mathrm{mL}$. However, the root bark extract showed weak cytotoxicity at $0.5 \mathrm{mg} / \mathrm{mL}$, and strong cytotoxicity at $2 \mathrm{mg} / \mathrm{mL}$. The strongest cytotoxicity of the root bark extract may come from the very large amount of dicaffeoylspermidines (kukoamine A/B and propionyl-dicaffeoylspermidine). To prove this hypothesis, the cytotoxicity of kukoamine B was tested. The results showed that kukoamine B (Figure $5 \mathrm{~b}$ ) had strong toxicity at $0.2 \mathrm{mg} / \mathrm{mL}$, which was equivalent to the level of kukoamine $\mathrm{B}$ in $\sim 6 \mathrm{mg} / \mathrm{mL}$ root bark extract. Considering the similar amount of kukoamine A and propionyl-dicaffeoylspermidine existed together with kukoamine B in root bark extract, the higher cytotoxicity of root bark extract could mainly due to the high content of dicaffeoylspermidine/spermine derivatives. Although glycoalkaloids were only detected in the fruit extract, the extremely low levels cannot cause cytotoxicity.
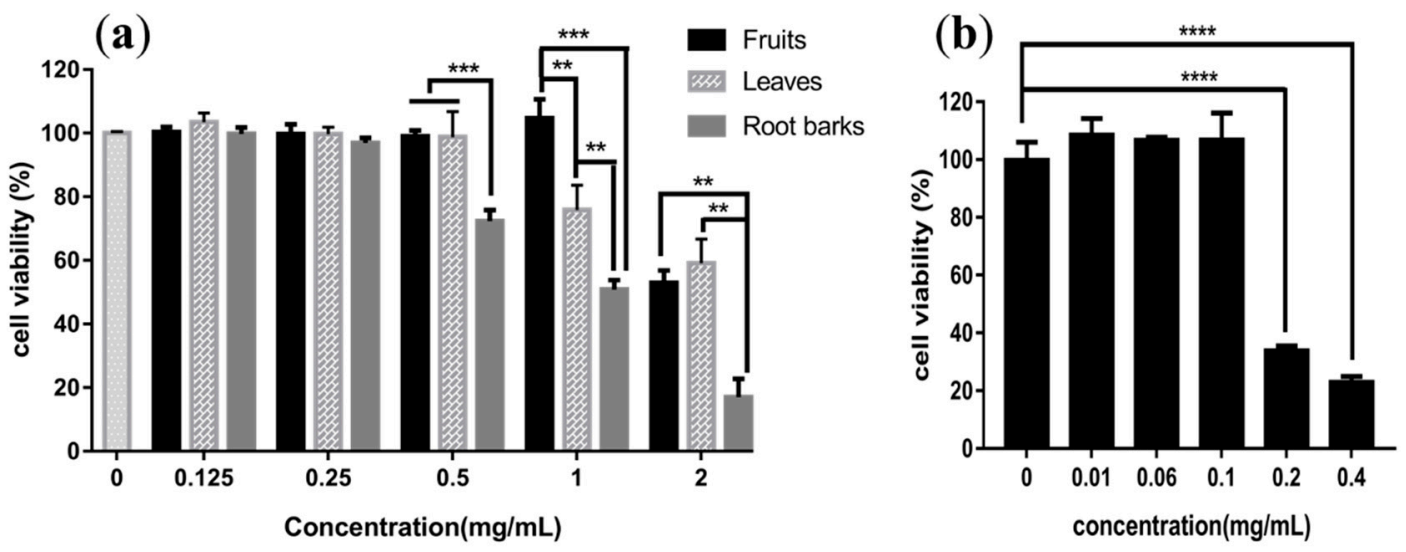

Figure 5. The cytotoxicities of different concentrations of extracts from fruits, leaves, root barks (a) and kukoamine B (b) towards L02 cells. Results were expressed as means \pm S.D., $n=3$. ${ }^{* *} p<0.01$, $* * * p<0.001, * * * * p<0.0001$.

\subsection{Potential Active Compounds Assay in Cells}

Since the fruit of L. barbarum is mostly used as a functional food and medicinal source, and contains much more components than leaves and root barks, the potential active compounds assay in the extracts will provide helpful information for understanding the action mechanism of fruits of L. barbarum. In general, to exert an effect, bioactive molecules should bind receptors or enzymes on cell membranes or enter into cells to interact with their molecular targets. Although a large number of compounds are presented in the extract of plant herbs, only a few of them can bind or enter into cells. Therefore, the cell-based screening has been applied for identification of potential bioactive components in plant herbs [53]. In order to identify the potential active compounds, a compound database of fruits of L. barbarum containing 91 chemicals was successfully established based on the quasimolecular ions in Q1 and the characteristic fragment ions in Q3 as ion pairs in MRM mode for the first time. The MRM ion pairs and corresponding declustering potential (DP) and collision energy (CE) of each constituent were optimized and presented in Table S4. 
To screen bioactive molecules, L02 cells were incubated with the fruit extracts for $4 \mathrm{~h}$ and then extracted with methanol. The methanol extract of cells was analyzed by UPLC-Qtrap-MS in MRM mode under the same condition with the construction of database. The typical total ion chromatography (TIC) of the extracts of L02 cells treated and untreated with fruit extracts are shown in Figure 6. More than 14 compounds were detected with the retention time in the range of 27-36 min, suggesting that the compounds entered cells have relatively high hydrophobicity. In total, at least eight dicaffeoylspermidine derivatives (e.g., B31 and B37) were detected in the cells. Two flavones (rutin (D5) and D6) were observed in L02 cells. Interestingly, three saponins (E2, E4 and E6) were detected in cells with higher relative content compared to that detected in the extract. Because E2 (solasonine) and E4 (5,6-dihydrosolasonine) were detected in the extract in a very low concentration, their observation in cells suggests that solasonine and 5,6-dihydrosolasonine were enriched in cells. These compounds detected in cells may have potential biological activities, which should be clarified in the future research.

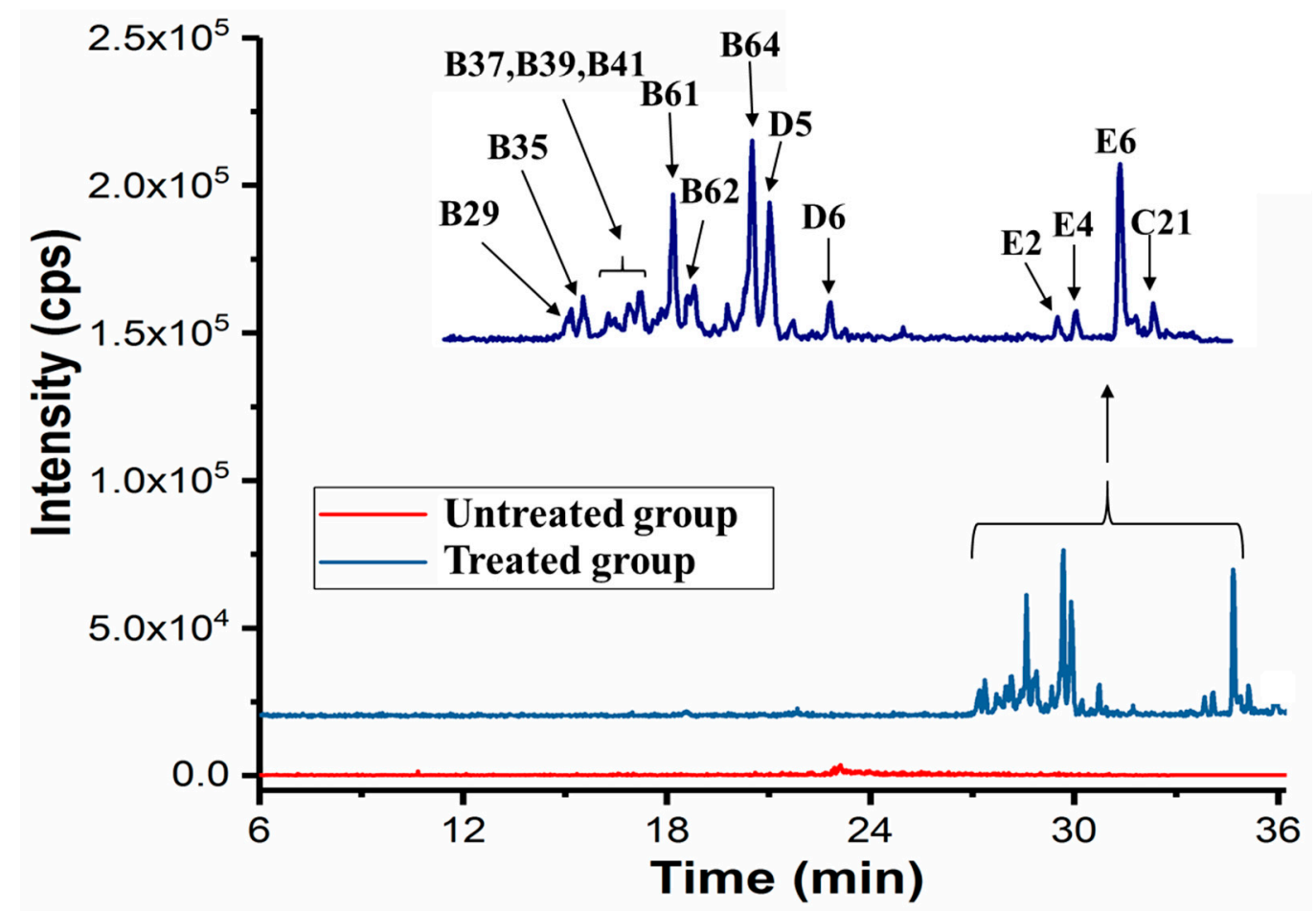

Figure 6. TIC of the extract of L02 cells treated and untreated with fruit extract. All the compound numbers were same with those shown in Table 1 and Table S4.

\section{Materials and Methods}

\subsection{Materials and Reagents}

The dried fruits, leaves and root barks of L. barbarum were purchased from Ningxia Province. Rutin, kukoamine B, chlorogenic acid, scopolin, solasonine and $\mathrm{N}$-p-trans-coumaroyltyramine were purchased from Baoji Herbest Bio-Tech Co., Ltd. (Baoji, China). 5,6-Dihydrosolasonine were isolated from the fruit of L. barbarum and identified by HR-ESI-MS and NMR techniques. Purities of all compounds were above $96 \%$ by HPLC analysis. HPLC grade methanol, acetonitrile, and MS grade formic acid were purchased from Thermo Fisher Scientific (Pittsburgh, PA, USA). Other chemicals and solvents were of analytical reagent grade.

L02 cells (normal human hepatic cell line) were obtained from National Experimental Cell Resource Sharing Platform (Beijing, China). Gentamicin, fetal bovine serum (FBS) were acquired from Gibco (Invitrogen, Paisley, UK). Dimethyl sulphoxide (DMSO) was purchased from Panreac (Barcelona, Spain). Dulbecco's modified Eagle's medium (DMEM), 2,2-diphenyl-1-picryl-hydrazyl (DPPH), hydrogen 
peroxide $(30 \% w / w), 2,4,6$-tris(2-pyridyl)-1,3,5-triazine (TPTZ), 2', $7^{\prime}$-dichlorodihydrofluorescein diacetate (DCFH-DA), ascorbic acid and 6-hydroxy-2,5,7,8-tetramethylchroman-2-carboxylic acid (Trolox) were obtained from Sigma-Aldrich (St. Louis, MO, USA).

\subsection{Sample Preparation}

For bioactivity analysis, the fruits, leaves and root barks (50 g of each) of L. barbarum were pulverized into powder and extracted thrice by ultrasound with ethanol/water (70:30, v:v) $(500 \mathrm{~mL}$, $1 \mathrm{~h} ; 400 \mathrm{~mL}, 1 \mathrm{~h} ; 400 \mathrm{~mL}, 1 \mathrm{~h}$ ), respectively. After filtration and freeze-drying, the crude products were $20.5 \mathrm{~g}, 14.3 \mathrm{~g}, 14.5 \mathrm{~g}$, respectively. The stock solutions of the extracts were prepared by dissolving the freeze-dried extracts in DMSO $(200 \mathrm{mg} / \mathrm{mL})$. For UPLC-MS analysis, the powder of fruits, leaves and root barks (100 mg of each) were extracted by ultrasound with $1 \mathrm{~mL}$ of ethanol/water (70:30, v:v) for $1 \mathrm{~h}$. After centrifugation, the supernatants were applied for UPLC-MS analysis.

\subsection{Isolation and Identification of 5,6-dihydrosolasonine from Fruit of L. barbarum}

The dried fruit of L. barbarum (5 kg) was extracted twice by ultrasound with $25 \mathrm{~L}$ of $70 \%$ ethanol/water for $1 \mathrm{~h}$ each time. After filtration, the ethanol was removed under reduced pressure to yield a concentrated solution. The solution was passed through a macroporous resin column (AB-8) and successively eluted with $0,20,80 \%$ ethanol/water. Finally, the $80 \%$ ethanol/water fraction was concentrated under reduced pressure, then extracted with butanol for three times and concentrated under vacuum. After dissolving this fraction with methanol, the target compounds were purified by preparative high performance liquid chromatography. The HR-ESI-MS data were recorded on a Orbitrap mass spectrometer (Thermo Fisher Scientific, Bremen, Germany). NMR spectra were acquired with Bruker AV 600 spectrometers (Bruker BioSpin Group, Faellanden, Switzerland) using the solvent signals (in $\mathrm{C}_{5} \mathrm{D}_{5} \mathrm{~N}$ ) as internal standards.

\subsection{Multiple Component Identification by UPLC-Orbitrap-MS}

The ultimate 3000 hyperbaric LC system coupled with high resolution Orbitrap Fusion Lumos Tribrid $^{\mathrm{TM}}$ via an electrospray ionization (ESI) interface (Thermo Fisher Scientific) was used for a comprehensive analysis of the constituents in fruit, leaf and root bark extracts. A BEH C18 column $\left(1.7 \mu \mathrm{m}, 2.1 \mathrm{~mm}\right.$ ID $\times 100 \mathrm{~mm}$, Waters, Milford, MA, USA) maintained at $35^{\circ} \mathrm{C}$ was finally chosen for separation of these extracts. The mobile phase was water $(0.1 \%$ formic acid, A) mixed in gradient mode with acetonitrile ( $0.1 \%$ formic acid, B), at a flow rate of $200 \mu \mathrm{L} / \mathrm{min}$. The elution gradient was optimized as follows: $0-3 \mathrm{~min}, 3 \% \mathrm{~B} ; 3-10 \mathrm{~min}, 3 \%$ to $5 \% \mathrm{~B} ; 10-25 \mathrm{~min}, 5 \%$ to $20 \% \mathrm{~B} ; 25-30 \mathrm{~min}, 20 \%$ to $50 \% \mathrm{~B} ; 30-33 \mathrm{~min}, 50 \%$ to $100 \% \mathrm{~B} ; 33-42 \mathrm{~min}, 100 \% \mathrm{~B}$. The injection volume was $3.0 \mu \mathrm{L}$ and samples were set at $4{ }^{\circ} \mathrm{C}$.

For identification of the components in the extracts, both positive and negative full scan modes within the mass/charge $(\mathrm{m} / \mathrm{z})$ ratio range of 150-1500 at a resolution of 120,000 were used for acquisition of accurate molecular ions. The other parameters were as follows: spray voltage, $+3.0 \mathrm{kV}$ in the positive mode and $-2.0 \mathrm{kV}$ in the negative mode; sheath gas flow rate, $35 \mathrm{Arb}$; aux gas flow rate, $10 \mathrm{Arb}$; sweep gas flow rate, $2 \mathrm{Arb}$; ion transfer tube temperature, $325^{\circ} \mathrm{C}$, vaporizer temperature, $275^{\circ} \mathrm{C}$. The fragment ions in MS/MS data obtained by higher energy collision dissociation (HCD, collision energy: $35 \mathrm{eV}$ ) were further utilized for confirmation of the structures of components. In addition, standards were also used for assistance of component identification. $\mathrm{X}_{\text {calibur }}{ }^{\mathrm{TM}} 3.0$ software was used for UPLC-HR-MS control and data handling.

\subsection{Compound Database Construction by UPLC-Qtrap-MS}

An AB Sciex Qtrap ${ }^{\circledR} 4500$ tandem MS (Foster City, CA, USA) equipped with an ESI source connected to the UPLC system (I-class Acquity UPLC, Waters, Framingham, MA, USA) was used to construct the compound database. Firstly, an instrument method in MRM $(\mathrm{Q} 1=\mathrm{Q} 3)$ information-dependent acquisition (IDA)-enhanced product ion (EPI) mode [18] for the analysis of fruit extract was established 
on the basis of the identified compounds from fruits by UPLC-HR-MS. Further, product ion scanning experiments were conducted and the DP and CE was optimized for each analyte to generate the most abundant product ions. The product ion spectra were further used to select the precursor-product ion pairs for the development of MRM assays. Finally, a compound database of fruit of L. barbarum was established based on the quasimolecular ion in Q1 and its characteristic fragment ions in Q3 as ion pair in MRM mode. This database was used for the next screening of active compounds.

\subsection{Quantitative Analysis of Compounds in Extracts}

UPLC-Qtrap-MS was used for quantitative analysis of seven compounds in the extracts of fruits, leaves and root barks by MRM mode. The liquid chromatographic conditions were the same as those of UPLC-Orbitrap-MS analysis. Method validation was carried out for seven standards in terms of linearity, sensitivity, intra/inter-day precisions and recovery [54]. The linearity was obtained by preparing a series of concentrations of standards solution with at least five appropriate concentrations in duplicate. The LOD and the LOQ for each analyte were acquired while the S/N was 3 and 10, respectively. The precision (inter and intra-day precision) was analyzed using the standard solutions with six replicates, and the RSD of the peak area for each standard was calculated. A spike recovery test was used to evaluate the accuracy of these methods. Three concentrations (high, middle and low) of mixed standard solutions were added to fruit extract respectively, then quantitative analysis was performed as described above. Each standard was tested at each concentration in triplicate. The spike recoveries were calculated using the following equation: Recovery $\%=[$ (measured amount-original amount)/amount added] $\times 100, \mathrm{RSD}=\mathrm{SD} /$ mean $\times 100$ [55]. Additionally, quantification of seven compounds using UPLC-Qtrap-MS was also established and the MRM pairs, DP and CE were optimized based on the standards.

\subsection{In Vitro Antioxidative Assays}

\subsubsection{DPPH Radical Scavenging Activity}

The effect of the extracts against 2,2-diphenyl-1-picrylhydrazyl (DPPH) radical was tested according to a previous report [56] with slight modifications. In brief, $100 \mu \mathrm{L}$ of $0.2 \mathrm{mM}$ DPPH radical solution in ethanol was added to $100 \mu \mathrm{L}$ of extract solutions at different concentrations. After incubation for $30 \mathrm{~min}$ at room temperature in the dark, the absorbance was read at $517 \mathrm{~nm}$. Ascorbic acid was used as a positive control and all measurements were done in triplicate. The extract concentration providing $50 \%$ inhibition $\left(\mathrm{IC}_{50}\right.$ ) was calculated by plotting inhibition percentages against the concentrations of extracts. The DPPH radical scavenging rate $(S \%)$ was calculated as follows: $S \%=\left[\left(A_{0}-A_{1}\right) / A_{0}\right] \times 100$ $\left(\mathrm{A}_{1}\right.$ and $\mathrm{A}_{0}$ are the absorbance of DPPH radical solution after incubation with and without extracts, respectively).

\subsubsection{ABTS Radical Scavenging Assay}

ABTS $^{\bullet+}$ scavenging activity was measured according to the defined method with slight modifications [57]. In brief, the radical cations were prepared by reacting $7 \mathrm{mM}$ aqueous ABTS with $2.45 \mathrm{mM}$ potassium persulphate. The mixture was allowed to stand in the dark at room temperature for $16 \mathrm{~h}$ before use and the $\mathrm{ABTS}^{\bullet+}$ solution was diluted with methanol to an absorbance of $0.700 \pm 0.020$ at $734 \mathrm{~nm}$. Different concentrations of extracts in methanol $(40 \mu \mathrm{L})$ were added to $160 \mu \mathrm{L}$ of $\mathrm{ABTS}^{\bullet+}$ solution and the absorbance was recorded after $4 \mathrm{~min}$. The $\mathrm{IC}_{50}$ and percentage inhibition of absorbance at $734 \mathrm{~nm}$ were calculated. All measurements were done in triplicate. Inhibition of ABTS ${ }^{\bullet+}$ in percent, $I(\%)$ was calculated as follows: $I(\%)=\left(A_{b}-A_{s} / A_{b}\right) \times 100$, where $A_{b}$ was the absorbance of the control and $\mathrm{A}_{\mathrm{S}}$ was the absorbance of tested samples. 


\subsubsection{FRAP Assay}

The principle of the FRAP assay is based on the reduction of ferric-tripyridyltriazine complex to its ferrous (colored form) in the presence of antioxidants. The FRAP assay was performed as described previously [58]. Briefly, the FRAP reagent contained $5 \mathrm{~mL}$ of $10 \mathrm{mM}$ TPTZ (2,4,6-tripyridy-s-triazine) solution in $40 \mathrm{mM} \mathrm{HCl}$ and $5 \mathrm{~mL}$ of $20 \mathrm{mM} \mathrm{FeCl}_{3} \cdot 6 \mathrm{H}_{2} \mathrm{O}$ in $300 \mathrm{mM}$ acetate buffer $(\mathrm{pH}=3.6)$. The mixture was freshly prepared and warmed at $37^{\circ} \mathrm{C}$ for $30 \mathrm{~min}$. In parallel, a solution containing $5 \mu \mathrm{L}$ of ultrapure water and $155 \mu \mathrm{L}$ of FRAP solution was prepared as the negative control. Different concentrations of extracts in methanol $(5 \mu \mathrm{L})$ were mixed with $155 \mu \mathrm{L}$ of FRAP solution and kept for $30 \mathrm{~min}$ in the dark. The ferrous tripyridyltriazine complex (coloured product) was measured by reading the absorbance at $593 \mathrm{~nm}$. The antioxidative capacity of test samples was given by the $\mathrm{RC}_{50}$ value, the concentration $(\mu \mathrm{g} / \mathrm{mL})$ necessary for a $50 \%$ reduction of $\mathrm{Fe}^{3+}$. Trolox was used as the positive control with a concentration ranging from 0 to $150 \mu \mathrm{g} / \mathrm{mL}$.

\subsection{Reactive Oxygen Species Measurement in L02 Cell}

In cells, reactive oxygen species (ROS) were determined using a fluorescent dye protocol [59]. Cells were treated with different concentrations of each extract for $1 \mathrm{~h}$ and then incubated with $\mathrm{H}_{2} \mathrm{O}_{2}$ $(100 \mu \mathrm{M})$ for $1 \mathrm{~h}$. The DCF fluorescence intensity was detected on a SpectraMax M5 microplate reader (Molecular Devices, Sunnyvale, CA, USA) with excitation at $488 \mathrm{~nm}$ and emission at $535 \mathrm{~nm}$. The confocal imaging was performed on an OLYMPUS FV3000-IX81 confocal microscope (Olympus Corporation, Tokyo, Japan). Confocal images were processed by Olympus FV10-ASW 4.2 viewer software (Olympus Corporation, Tokyo, Japan).

\subsection{Cytotoxicity Assay}

L02 cells were cultured in 10\% FBS-supplemented DMEM and 1\% gentamicin, and kept in a humidified atmosphere of $5 \% \mathrm{CO}_{2}$ at $37^{\circ} \mathrm{C}$. For cytotoxicity assay, L02 cells in logarithmic growth phase were plated in 96-well plates at a density of $5 \times 10^{3}$ cells per well in $100 \mu \mathrm{L}$ of culture medium and were allowed to adhere for $24 \mathrm{~h}$ before treatment. Serial concentrations of each sample (fruit, leaf and root bark extracts and kukoamine B) were then added (100 $\mu \mathrm{L}$ per well). After treated for $24 \mathrm{~h}, 10 \mu \mathrm{L}$ of CCK-8 solution was added to each well and incubated at $37^{\circ} \mathrm{C}, 5 \% \mathrm{CO}_{2}$ for $1 \mathrm{~h}$. The absorbance at $450 \mathrm{~nm}$ was measured using a SpectraMax M5 microplate reader (Molecular Devices).

\subsection{Target Cell-Based Screening of Potential Active Compounds}

The target cell-based screening of potential active compounds in the fruit of L. barbarum was performed as described in previous study with slight modifications [15]. Specifically, L02 cells in the logarithmic growth phase were seeded into cell culture dish at a density of $1.0 \times 10^{6} \mathrm{cells} / \mathrm{mL}$, and were cultured in DMEM medium at $37^{\circ} \mathrm{C}$ for $24 \mathrm{~h}$. The culture medium was replaced by $3 \mathrm{~mL}$ of fruits extract of L. barbarum diluted in DMEM (free of serum) at a final concentration of $10 \mathrm{mg} / \mathrm{mL}$, and incubated at $37^{\circ} \mathrm{C}$ for $4 \mathrm{~h}$. The incubation solution was discarded and the cells were washed five times with phosphate-buffered saline to remove free components. Finally, the cells were collected and extracted with $200 \mu \mathrm{L}$ of methanol and centrifuged at 12,000 rpm for $10 \mathrm{~min}$. The obtained supernatant was used for UPLC-Qtrap-MS analysis. The control sample without extract treatment was prepared by the same procedures as above.

\subsection{Data Handling and Presentation/Statistical Analysis}

Data for quantification were acquired from individual experiments repeated at least three times, and expressed as the means \pm SD. Statistical significance was calculated by GraphPad Prism 6 software (GraphPad Software, Inc., San Diego, CA, USA) with unpaired two-tailed t-tests and accepted by $\left.\left.p<0.05\left(^{*}\right), p<0.01{ }^{(* *}\right), p<0.0011^{(* *}\right), p<0.0001^{(* * *)}$. The $\mathrm{IC}_{50}$ or $\mathrm{RC}_{50}$ were calculated using the 
GraphPad Prism 6 software according to the inhibition rates or reduction rates (y) plotted against the sample concentrations $(\mathrm{x})$.

\section{Conclusions}

In this study, a total of 131 compounds were identified in extracts (70\% ethanol) from fruits, leaves and root barks of L. barbarum by UPLC-Orbitrap-MS and seven of them were quantified by UPLC-Qtrap-MS. The distribution of these compounds in the three parts of L. barbarum was significantly different. The fruit extract contained the most compounds. A very large amount of kukoamine A/B (dicaffeoylspermine isomers) and propionyl-dicaffeoylspermidine were found in the extracts of root barks, and a high amount of dicaffeoylspermidine isomers were detected in the fruits and leaves. Many glycosylated derivatives of dicaffeoylspermine/dicaffeoylspermidine were mainly detected in the fruits. The bioactivity assays showed that the fruit extracts had the lowest antioxidant activity and cytotoxicity. The root bark extracts showed the strongest antioxidant activity and cytotoxicity, which was caused by the large amount of dicaffeoylspermidine/spermine derivatives. Six saponins were found in L. barbarum plants for the first time, and they were only detected in the fruits; among them, 5,6-dihydrosolasonine, a new glycoside alkaloid (saponin) was isolated and characterized. In addition, 14 potential bioactive compounds were detected in L02 cells after treated with the extracts of fruits. All these results will provide important information for understanding the different biological activities of the three parts of L. barbarum plants and will be beneficial for drug discovery from L. barbarum plants.

Supplementary Materials: The following are available online, Figure S1: Total ion chromatograms (TICs) of 7 standard substances in the positive ion mode; Figure S2: Proposed fragmentation pathway for solasonine, based on HR-Orbitrap MS/MS spectra; Figure S3. The HR-Orbitrap MS/MS spectra and Proposed fragmentation pathway of chlorogenic acid; Figure S4. The HR-Orbitrap MS/MS spectra and proposed fragmentation pathway of scopolin; Figure S5. The HR-Orbitrap MS/MS spectrum and the proposed fragmentation pathway of kukoamine B; Figure S6. The HR-Orbitrap MS/MS spectrum and the proposed fragmentation pathway of N-p-trans-Coumaroyl tyramine; Figure S7. The HR-Orbitrap MS/MS spectrum and the proposed fragmentation pathway of rutin; Figure S8. HR-Orbitrap MS/MS spectrum of A1; Figure S9. HR-Orbitrap MS/MS spectrum of B52; Figure S10. HR-Orbitrap MS/MS spectra of B63; Figure S11. HR-Orbitrap MS/MS spectrum of B18; Figure S12. HR-Orbitrap MS/MS spectra of compounds B46, B50 and B54; Figure S13. HR-Orbitrap MS/MS spectra of B51; Figure S14. HR-Orbitrap MS/MS spectra of C15; Figure S15. HR-Orbitrap MS/MS spectrum of D6; Figure S16. HR-Orbitrap MS/MS spectrum of E3; Figure S17. Protection Effect of the extracts from fruits $(0.25 \mathrm{mg} / \mathrm{mL})$, leaves and root barks $(0.2 \mathrm{mg} / \mathrm{mL})$ on $100 \mu \mathrm{M} \mathrm{H}_{2} \mathrm{O}_{2}$-induced intracellular ROS production in L02 cells. Table S1.13C NMR spectral data for aglycone and glycoside moieties of solasonine (SS) and 5.6-dihydrosolasonine (2HSS) in pyridine (D5); Table S2. The regression equation, LOD, LOQ, intra-day and inter-day of the 7 standards using the optimized method for calibration; Table S3. The recoveries of 7 standards $(n=3)$; Table S4. MS/MS parameters for the construction of the database of the 91 compounds in fruit of L. barbarum using UPLC- Qtrap-MS in the positive ion mode.

Author Contributions: X.X., W.R., Z.Z. and D.S. designed the experiment; X.X., W.R. performed the experiments with the help of N.Z., T.B., X.L. and X.X., W.R., D.S. and Z.Z. analyzed the data; X.X., D.S., W.R., and Z.Z. wrote the paper; D.S. conceived and directed the overall project. All authors have given approval to the final version of the manuscript.

Funding: This research was funded by NSF of China, grant number 21635008, 21877115, 21874140, 21575147 and 21621062.

Conflicts of Interest: The authors would like to declare no conflict of interest in the publication of this research.

\section{References}

1. Wang, K.; Sasaki, T.; Li, W.; Li, Q.; Wang, Y.; Asada, Y.; Kato, H.; Koike, K. Two novel steroidal alkaloid glycosides from the seeds of Lycium barbarum. Chem. Biodivers. 2011, 8, 2277-2284. [CrossRef]

2. Wang, Y.; Zhao, B.; Ma, H.R.; Aisa, H.A. Two new sesquiterpenoid glycosides from the leaves of Lycium barbarum. J. Asian Nat. Prod. Res. 2016, 18, 871-877. [CrossRef]

3. Potterat, O. Goji (Lycium barbarum and L. chinense): Phytochemistry, pharmacology and safety in the perspective of traditional uses and recent popularity. Planta Med. 2010, 76, 7-19. [CrossRef]

4. Yu, M.S.; Leung, S.K.; Lai, S.W.; Che, C.M.; Zee, S.Y.; So, K.F.; Yuen, W.H.; Chang, R.C. Neuroprotective effects of anti-aging oriental medicine Lycium barbarum against beta-amyloid peptide neurotoxicity. Exp. Gerontol. 2005, 40, 716-727. [CrossRef] 
5. Amagase, H.; Farnsworth, N.R. A review of botanical characteristics, phytochemistry, clinical relevance in efficacy and safety of Lycium barbarum fruit (Goji). Food Res. Int. 2011, 44, 1702-1717. [CrossRef]

6. Dong, J.Z.; Gao, W.S.; Lu, D.Y.; Wang, Y. Simultaneous Extraction and Analysis of Four Polyphenols from Leaves of Lycium Barbarum L. J. Food Biochem. 2011, 35, 914-931. [CrossRef]

7. Yang, Y.; An, Y.; Wang, W.; Du, N.; Zhang, J.; Feng, Z.; Jiang, J.; Zhang, P. Nine compounds from the root bark of Lycium chinense and their anti-inflammatory activitieslammatory activitiesretain. Acta Pharm. Sin. B 2017, 7, 491-495. [CrossRef] [PubMed]

8. Zhang, J.; Guan, S.; Sun, J.; Liu, T.; Chen, P.; Feng, R.; Chen, X.; Wu, W.; Yang, M.; Guo, D.A. Characterization and profiling of phenolic amides from Cortex Lycii by ultra-high performance liquid chromatography coupled with LTQ-Orbitrap mass spectrometry. Anal. Bioanal. Chem. 2015, 407, 581-595. [CrossRef]

9. Qian, D.; Zhao, Y.; Yang, G.; Huang, L. Systematic Review of Chemical Constituents in the Genus Lycium (Solanaceae). Molecules 2017, 22, 911. [CrossRef]

10. Zhang, J.X.; Guan, S.H.; Feng, R.H.; Wang, Y.; Wu, Z.Y.; Zhang, Y.B.; Chen, X.H.; Bi, K.S.; Guo, D.A. Neolignanamides, lignanamides, and other phenolic compounds from the root bark of Lycium chinense. J. Nat. Prod. 2013, 76, 51-58. [CrossRef] [PubMed]

11. Mocan, A.; Vlase, L.; Vodnar, D.C.; Bischin, C.; Hanganu, D.; Gheldiu, A.M.; Oprean, R.; Silaghi-Dumitrescu, R.; Crisan, G. Polyphenolic content, antioxidant and antimicrobial activities of Lycium barbarum L. and Lycium chinense Mill. leaves. Molecules 2014, 19, 10056-10073. [CrossRef]

12. Panidthananon, W.; Chaowasku, T.; Sritularak, B.; Likhitwitayawuid, K. A New Benzophenone C-Glucoside and Other Constituents of Pseuduvaria fragrans and Their alpha-Glucosidase Inhibitory Activity. Molecules 2018, 23, 1600. [CrossRef]

13. Inbaraj, B.S.; Lu, H.; Kao, T.H.; Chen, B.H. Simultaneous determination of phenolic acids and flavonoids in Lycium barbarum Linnaeus by HPLC-DAD-ESI-MS. J. Pharm. Biomed. Anal. 2010, 51, 549-556. [CrossRef] [PubMed]

14. Mocan, A.; Vlase, L.; Raita, O.; Hanganu, D.; Paltinean, R.; Dezsi, S.; Gheldiu, A.M.; Oprean, R.; Crisan, G. Comparative studies on antioxidant activity and polyphenolic content of Lycium barbarum L. and Lycium chinense Mill. leaves. Pak. J. Pharm. Sci. 2015, 28, 1511-1515.

15. Ren, W.; Han, L.; Luo, M.; Bian, B.; Guan, M.; Yang, H.; Han, C.; Li, N.; Li, T.; Li, S.; et al. Multi-component identification and target cell-based screening of potential bioactive compounds in toad venom by UPLC coupled with high-resolution LTQ-Orbitrap MS and high-sensitivity Qtrap MS. Anal. Bioanal. Chem. 2018, 410, 4419-4435. [CrossRef] [PubMed]

16. Patras, M.A.; Jaiswal, R.; McDougall, G.J.; Kuhnert, N. Profiling and Quantification of Regioisomeric Caffeoyl Glucoses in Berry Fruits. J. Agric. Food Chem. 2018, 66, 1096-1104. [CrossRef] [PubMed]

17. Mocan, A.; Moldovan, C.; Zengin, G.; Bender, O.; Locatelli, M.; Simirgiotis, M.; Atalay, A.; Vodnar, D.C.; Rohn, S.; Crisan, G. UHPLC-QTOF-MS analysis of bioactive constituents from two Romanian Goji (Lycium barbarum L.) berries cultivars and their antioxidant, enzyme inhibitory, and real-time cytotoxicological evaluation. Food Chem. Toxicol. 2018, 115, 414-424. [CrossRef] [PubMed]

18. Guan, M.; Dai, D.; Li, L.; Wei, J.; Yang, H.; Li, S.; Zhang, Y.; Lin, Y.; Xiong, S.; Zhao, Z. Comprehensive qualification and quantification of triacylglycerols with specific fatty acid chain composition in horse adipose tissue, human plasma and liver tissue. Talanta 2017, 172, 206-214. [CrossRef]

19. Lelario, F.; Labella, C.; Napolitano, G.; Scrano, L.; Bufo, S.A. Fragmentation study of major spirosolane-type glycoalkaloids by collision-induced dissociation linear ion trap and infrared multiphoton dissociation Fourier transform ion cyclotron resonance mass spectrometry. Rapid Commun. Mass Spectrom. 2016, 30, 2395-2406. [CrossRef]

20. Lee, Y.-Y.; Hashimoto, F.; Yahara, S.; Nohara, T.; Yoshida, N. Study on the solanacenous plants. Part 29. Steroidal Glycosides from Solanum dulcamara. Chem. Pharm. Bull. 1994, 42, 707-709. [CrossRef]

21. Matsushita, S.; Yanai, Y.; Fusyuku, A.; Ikeda, T.; Ono, M.; Nohara, T. Distinction of absolute configuration at C-22 of C-23-hydroxyspirostane and C-23-hydroxyspirosolane glycosides. Chem. Pharm. Bull. 2007, 55, 1079-1081. [CrossRef]

22. Al Sinani, S.S.S.; Eltayeb, E.A. The steroidal glycoalkaloids solamargine and solasonine in Solanum plants. South Afr. J. Bot. 2017, 112, 253-269. [CrossRef]

23. Trivedi, P.; Pundarikakshudu, K.J.C. Novel TLC Densitometric Method for Quantification of Solasodine in Various Solanum Species, Market Samples and Formulations. Chromatographia 2007, 65, 239-243. [CrossRef] 
24. Dinan, L.; Harmatha, J.; Lafont, R. Chromatographic procedures for the isolation of plant steroids. J. Chromatogr. A 2001, 935, 105-123. [CrossRef]

25. Milner, S.E.; Brunton, N.P.; Jones, P.W.; O’Brien, N.M.; Collins, S.G.; Maguire, A.R. Bioactivities of glycoalkaloids and their aglycones from Solanum species. J. Agric. Food Chem. 2011, 59, 3454-3484. [CrossRef] [PubMed]

26. Weissenberg, M. Isolation of solasodine and other steroidal alkaloids and sapogenins by direct hydrolysis-extraction of Solanum plants or glycosides therefrom. Phytochemistry 2001, 58, 501-508. [CrossRef]

27. Roddick, J.G.; Weissenberg, M.; Leonard, A.L. Membrane disruption and enzyme inhibition by naturally-occurring and modified chacotriose-containing Solanum steroidal glycoalkaloids. Phytochemistry 2001, 56, 603-610. [CrossRef]

28. Zhou, Z.Q.; Xiao, J.; Fan, H.X.; Yu, Y.; He, R.R.; Feng, X.L.; Kurihara, H.; So, K.F.; Yao, X.S.; Gao, H. Polyphenols from wolfberry and their bioactivities. Food Chem. 2017, 214, 644-654. [CrossRef] [PubMed]

29. Wolfram, K.; Schmidt, J.; Wray, V.; Milkowski, C.; Schliemann, W.; Strack, D. Profiling of phenylpropanoids in transgenic low-sinapine oilseed rape (Brassica napus). Phytochemistry 2010, 71, 1076-1084. [CrossRef] [PubMed]

30. Funayama, S.; Zhang, G.-R.; Nozoe, S.; Kukoamine, B. A spermine alkaloid from Lycium chinense. Phytochemistry 1995, 38, 1529-1531. [CrossRef]

31. Li, Y.Y.; Wang, H.; Zhao, C.; Huang, Y.Q.; Tang, X.; Cheung, H.Y. Identification and Characterization of Kukoamine Metabolites by Multiple Ion Monitoring Triggered Enhanced Product Ion Scan Method with a Triple-Quadruple Linear Ion Trap Mass Spectrometer. J. Agric. Food Chem. 2015, 63, 10785-10790. [CrossRef] [PubMed]

32. Zhou, Z.Q.; Fan, H.X.; He, R.R.; Xiao, J.; Tsoi, B.; Lan, K.H.; Kurihara, H.; So, K.F.; Yao, X.S.; Gao, H. Lycibarbarspermidines A-O, New Dicaffeoylspermidine Derivatives from Wolfberry, with Activities against Alzheimer's Disease and Oxidation. J. Agric. Food Chem. 2016, 64, 2223-2237. [CrossRef] [PubMed]

33. Yossa Nzeuwa, I.B.; Xia, Y.; Qiao, Z.; Feng, F.; Bian, J.; Liu, W.; Qu, W. Comparison of the origin and phenolic contents of Lycium ruthenicum Murr. by high-performance liquid chromatography fingerprinting combined with quadrupole time-of-flight mass spectrometry and chemometrics. J. Sep. Sci. 2017, 40, 1234-1243. [CrossRef] [PubMed]

34. Zhao, J.; Xu, F.; Ji, T.; Li, J. A New Spermidine from the Fruits of Lycium ruthenicum. Chem. Nat. Compounds 2014, 50, 880-883. [CrossRef]

35. Narváez-Cuenca, C.-E.; Vincken, J.-P.; Gruppen, H. Identification and quantification of (dihydro) hydroxycinnamic acids and their conjugates in potato by UHPLC-DAD-ESI-MSn. Food Chem. 2012, 130, 730-738. [CrossRef]

36. Seca, A.M.; Silva, A.M.; Silvestre, A.J.; Cavaleiro, J.A.; Domingues, F.M.; Pascoal-Neto, C. Lignanamides and other phenolic constituents from the bark of kenaf (Hibiscus cannabinus). Phytochemistry 2001, 58, 1219-1223. [CrossRef]

37. Strehmel, N.; Bottcher, C.; Schmidt, S.; Scheel, D. Profiling of secondary metabolites in root exudates of Arabidopsis thaliana. Phytochemistry 2014, 108, 35-46. [CrossRef] [PubMed]

38. Yang, L.; Jiang, H.; Wang, Q.-H.; Yang, B.-Y.; Kuang, H.-X. A new feruloyl tyramine glycoside from the roots of Achyranthes bidentata. Chin. J. Nat. Med. 2012, 10, 16-19. [CrossRef]

39. Gao, K.; Ma, D.; Cheng, Y.; Tian, X.; Lu, Y.; Du, X.; Tang, H.; Chen, J. Three New Dimers and Two Monomers of Phenolic Amides from the Fruits of Lycium barbarum and Their Antioxidant Activities. J. Agric. Food Chem. 2015, 63, 1067-1075. [CrossRef] [PubMed]

40. Ma, J.; Jones, S.H.; Hecht, S.M. Phenolic acid amides: a new type of DNA strand scission agent from Piper caninum. Bioorg. Med. Chem. 2004, 12, 3885-3889. [CrossRef]

41. Sun, J.; Gu, Y.F.; Su, X.Q.; Li, M.M.; Huo, H.X.; Zhang, J.; Zeng, K.W.; Zhang, Q.; Zhao, Y.F.; Li, J.; et al. Anti-inflammatory lignanamides from the roots of Solanum melongena L. Fitoterapia 2014, 98, 110-116. [CrossRef]

42. Mocan, A.; Zengin, G.; Simirgiotis, M.; Schafberg, M.; Mollica, A.; Vodnar, D.C.; Crisan, G.; Rohn, S. Functional constituents of wild and cultivated Goji (L. barbarum L.) leaves: Phytochemical characterization, biological profile, and computational studies. J. Enzyme. Inhib. Med. Chem. 2017, 32, 153-168. [CrossRef] [PubMed] 
43. Kang, K.B.; Ryu, J.; Cho, Y.; Choi, S.Z.; Son, M.; Sung, S.H. Combined Application of UHPLC-QTOF/MS, HPLC-ELSD and (1) H-NMR Spectroscopy for Quality Assessment of DA-9801, A Standardised Dioscorea Extract. Phytochem. Anal. 2017, 28, 185-194. [CrossRef]

44. Challinor, V.L.; Parsons, P.G.; Chap, S.; White, E.F.; Blanchfield, J.T.; Lehmann, R.P.; De Voss, J.J. Steroidal saponins from the roots of Smilax sp.: structure and bioactivity. Steroids 2012, 77, 504-511. [CrossRef] [PubMed]

45. Ji, D.; Su, X.; Huang, Z.; Su, L.; Li, L.; Lu, T. Analysis of Chemical Variations between Crude and Salt-Processed Anemarrhenae rhizoma Using Ultra-High-Performance Liquid Chromatography-Mass Spectrometry Methods. Molecules 2017, 23, 23. [CrossRef] [PubMed]

46. Ledesma-Escobar, C.A.; Priego-Capote, F.; Luque de Castro, M.D. Characterization of lemon (Citrus limon) polar extract by liquid chromatography-tandem mass spectrometry in high resolution mode. J. Mass Spectrom. 2015, 50, 1196-1205. [CrossRef] [PubMed]

47. Kokotkiewicz, A.; Migas, P.; Stefanowicz, J.; Luczkiewicz, M.; Krauze-Baranowska, M. Densitometric TLC analysis for the control of tropane and steroidal alkaloids in Lycium barbarum. Food Chem. 2017, 221, 535-540. [CrossRef]

48. Shanker, K.; Gupta, S.; Srivastava, P.; Srivastava, S.K.; Singh, S.C.; Gupta, M.M. Simultaneous determination of three steroidal glycoalkaloids in Solanum xanthocarpum by high performance thin layer chromatography. J. Pharm. Biomed. Anal. 2011, 54, 497-502. [CrossRef] [PubMed]

49. Affes, M.; Fakhfakh, J.; Daoud, I.; Brieudes, V.; Halabalaki, M.; El Feki, A.; Allouche, N. UHPLC/HR-ESI-MS/MS Profiling of Phenolics from Tunisian Lycium arabicum Boiss. Antioxidant and Anti-lipase Activities' Evaluation. Chem. Biodivers. 2017, 14. [CrossRef] [PubMed]

50. Luo, Q.; Cai, Y.; Yan, J.; Sun, M.; Corke, H. Hypoglycemic and hypolipidemic effects and antioxidant activity of fruit extracts from Lycium barbarum. Life Sci. 2004, 76, 137-149. [CrossRef] [PubMed]

51. Li, X.; Lin, J.; Chen, B.; Xie, H.; Chen, D. Antioxidant and Cytoprotective Effects of Kukoamines A and B: Comparison and Positional Isomeric Effect. Molecules 2018, 23, 973. [CrossRef]

52. Friedman, M. Chemistry and anticarcinogenic mechanisms of glycoalkaloids produced by eggplants, potatoes, and tomatoes. J. Agric. Food Chem. 2015, 63, 3323-3337. [CrossRef]

53. Hong, M.; Wang, X.Z.; Wang, L.; Hua, Y.Q.; Wen, H.M.; Duan, J.A. Screening of immunomodulatory components in Yu-ping-feng-san using splenocyte binding and HPLC. J. Pharm. Biomed. Anal. 2011, 54, 87-93. [CrossRef]

54. Song, Y.; Song, Q.; Liu, Y.; Li, J.; Wan, J.B.; Wang, Y.; Jiang, Y.; Tu, P. Integrated work-flow for quantitative metabolome profiling of plants, Peucedani Radix as a case. Anal. Chim. Acta 2017, 953, 40-47. [CrossRef]

55. Chen, S.; Liu, J.Q.; Xiao, H.; Zhang, J.; Liu, A. Simultaneous Qualitative Assessment and Quantitative Analysis of Metabolites (Phenolics, Nucleosides and Amino Acids) from the Roots of Fresh Gastrodia elata Using UPLC-ESI-Triple Quadrupole Ion MS and ESI- Linear Ion Trap High-Resolution MS. PLoS ONE 2016, 11. [CrossRef]

56. Li, Q.; Chen, J.; Li, T.; Liu, C.; Liu, W.; Liu, J. Comparison of bioactivities and phenolic composition of Choerospondias axillaris peels and fleshes. J. Sci. Food Agric. 2016, 96, 2462-2471. [CrossRef]

57. Can-Cauich, C.A.; Sauri-Duch, E.; Moo-Huchin, V.M.; Betancur-Ancona, D.; Cuevas-Glory, L.F. Effect of extraction method and specie on the content of bioactive compounds and antioxidant activity of pumpkin oil from Yucatan, Mexico. Food Chem. 2019, 285, 186-193. [CrossRef]

58. Benzie, I.F.; Strain, J.J. The ferric reducing ability of plasma (FRAP) as a measure of "antioxidant power": The FRAP assay. Anal. Biochem. 1996, 239, 70-76. [CrossRef]

59. Wolfe, K.L.; Liu, R.H. Cellular antioxidant activity (CAA) assay for assessing antioxidants, foods, and dietary supplements. J. Agric. Food Chem. 2007, 55, 8896-8907. [CrossRef]

Sample Availability: Not available.

(C) 2019 by the authors. Licensee MDPI, Basel, Switzerland. This article is an open access article distributed under the terms and conditions of the Creative Commons Attribution (CC BY) license (http://creativecommons.org/licenses/by/4.0/). 